\title{
Local Properties of Coulombic Wave Functions
}

\author{
M. Hoffmann-Ostenhof ${ }^{1}$, T. Hoffmann-Ostenhof ${ }^{2,3}$, H. Stremnitzer ${ }^{4}$ \\ 1 Institut für Mathematik, Universität Wien, Strudlhofgasse 4, A-1090 Wien, Austria \\ 2 Erwin Schrödinger International Institute of Mathematical Physics, Pasteurgasse 6/7, A-1090 Wien, \\ Austria \\ 3 Institut für Theoretische Chemie, Universität Wien, Währingerstrasse 17, A-1090 Wien, Austria \\ 4 Institut für Theoretische Physik, Universität Wien, Boltzmanngasse 5, A-1090 Wien, Austria
}

Received: 16 July 1993

\begin{abstract}
We investigate the local behaviour of solutions of a nonrelativistic Schrödinger equation which describe Coulombic systems. Firstly we give a representation theorem for such solutions in the neighbourhood of Coulombic singularities generalizing previous results (Cusp conditions) due to Kato and others. Secondly we investigate the influence of Fermi statistics on the local behaviour of many fermionic wave functions, showing that e.g. an $N$-electron wave function must have zeros of order at least $N^{4 / 3}$ for large $N$.
\end{abstract}

\section{Introduction}

In a recent paper [6] the local behaviour of a real valued local solution $u$ to the Schrödinger equation

$$
(-\Delta+V) u=0, \quad x \in \Omega, \quad \Omega \subseteq \mathbf{R}^{n}, \quad n \geq 3
$$

under rather weak assumptions on the real valued potential $V$ was investigated. It was shown that in the neighbourhood of a point $x_{0} \in \Omega$, assuming that $u$ vanishes there at most polynomially that

$$
u(x)=P_{M}\left(x-x_{0}\right)+\Phi\left(x-x_{0}\right),
$$

where $P_{M}(x) \not \equiv 0$ is a harmonic homogeneous polynomial of degree $M$ and $\Phi(x)=o\left(|x|^{M}\right)$. (See Sect. 1 for a precise statement.)

The purpose of the present paper is twofold. Firstly we give a detailed account of the local behaviour of solutions to the Schrödinger equation for Coulombic systems, e.g. atoms and molecules, especially in the neighbourhood of the singularities of the potential. Secondly (see Sect. 5) we investigate the influences of Fermi statistics on the local behaviour of many particle Fermionic wave functions. These results are consequences of (0.2) and some symmetry considerations and are not tied only to the Coulombic case.

Some of our results were recently announced in [7]. 


\section{Preliminaries and Statement of the Results}

We consider Coulombic Hamiltonians of $N$-particle systems with Hamiltonian

$$
H=-\sum_{i=1}^{N} \frac{\Delta_{\imath}}{2 m_{\imath}}+V_{C}\left(\vec{r}_{1}, \ldots, \vec{r}_{N}\right),
$$

where $\vec{r}_{\imath} \in \mathbf{R}^{3}$ denotes the position of the $i^{\text {th }}$ particle, $m_{i}>0$, denotes its mass and $\Delta_{i}$ denotes the 3-dimensional Laplacian with respect to the coordinates of the $i^{\text {th }}$ particle. The potential

$$
V_{C}\left(\vec{r}_{1} \ldots \vec{r}_{N}\right)=\sum_{i<j}^{N} \frac{\alpha_{i} \alpha_{j}}{\left|\vec{r}_{\imath}-\vec{r}_{\jmath}\right|}
$$

where the $\alpha_{i} \neq 0, i=1, \ldots, N$ denote the charges of the particles.

We shall describe the local behaviour of real valued solutions $\psi$ of the Schrödinger equation $H \psi=E \psi$, where $E \in \mathbf{R}$, in the neighbourhood of coalescence points (CP), where the potential $V_{C}$ is unbounded. Let $p\left(\vec{r}_{1}, \ldots, \vec{r}_{N}\right)=\prod_{i<j}\left|\vec{r}_{i}-\vec{r}_{j}\right|$. A point $\vec{r}^{\mathrm{CP}}:=\left(\vec{r}_{1}^{\mathrm{CP}}, \ldots, \vec{r}_{N}^{\mathrm{CP}}\right) \in \mathbf{R}^{d}, d=3 N$, is a $\mathrm{CP}$ if $p\left(\vec{r}_{1}^{\mathrm{CP}}, \ldots, \vec{r}_{N}^{\mathrm{CP}}\right)=0$. Obviously $V_{C}$ is real analytic away from the coalescence points and hence any local solution $\psi$ is real analytic there by elliptic regularity.

Before we can state our results on Coulombic systems we have to recall some notation and a result obtained in [6].

Let $\Omega$ be an open set in $\mathbf{R}^{d}, d \geq 3$ and let $V \in L_{\text {loc }}^{1}(\Omega)$ be a real valued function. We require that

$$
\begin{aligned}
& V \in K^{d, \delta}(\Omega) \text { for some } \delta>0 \text {, that means (compare [17, 5]) that } \\
& \qquad \lim _{\varepsilon \downarrow 0} \sup _{x \in \mathbf{R}^{d}} \int_{|x-y|<\varepsilon} \chi_{\Omega}(y) \frac{|V(y)|}{|x-y|^{d-2+\delta}} d y=0,
\end{aligned}
$$

where $\chi_{\Omega}$ denotes the characteristic function of $\Omega$. We consider a real valued distributional solution $u \neq \equiv 0$ of the Schrödinger equation $(-\Delta+V) u=0$ in $\Omega$,

$$
\text { so that } \forall \chi \in C_{0}^{\infty}(\Omega), \quad \int u(-\Delta+V) \chi d x=0 \text {. }
$$

The assumption on $V$ is well known (see [17] for a detailed discussion). For $\delta=0$ (A.1) defines the Kato class $K^{d}$ first introduced by Kato [12]. For $\delta>0$ it was shown that a solution of (1.3) is locally Hölder continuous [2,17]. Now let $u$ be a real valued solution to (1.3) and assume without loss that we consider the local behaviour near the origin $O$ and that $\Omega=B_{R}$, where $B_{R}=\left\{x \in \mathbf{R}^{d}:|x|<R\right\}$, for $R>0$ sufficiently small. We further assume that

$$
u \in C^{0}\left(B_{R}\right) \cap W^{1,2}\left(B_{R}\right),
$$

where $W^{1,2}$ denotes the usual Sobolev space. Moreover we assume that $u$ vanishes at most polynomially in $\mathbf{0}$, i.e.

$$
\sup _{\gamma \geq 0}\left\{\varlimsup_{r \downarrow 0} r^{-2 \gamma-d} \int_{B_{r}} u^{2}(y) d y<\infty\right\}<\infty .
$$


One of the main results in [6] on which our present investigations of Coulombic systems are based on is

Theorem 1.0. Let

$$
V \in K^{d, \delta}\left(B_{R}\right) \text { for some } \delta>0,
$$

and let $u \not \equiv 0$ be a real valued solution of (1.3) in $B_{R}$ satisfying (1.4) and (1.5). Then there exists a harmonic homogeneous polynomial $P_{M} \neq \equiv 0$ of degree $M \in \mathbf{N}_{0}$ such that

$$
u=P_{M}+\Phi \text { with } \Phi=O\left(|x|^{M+\min \left(1, \delta^{\prime}\right)}\right) \text { for }|x| \rightarrow 0, \text { for } \quad \delta^{\prime}<\delta .
$$

Let us first note that $V_{C}$ given in (1.2) satisfies (1.6), in fact (see [17])

$$
V_{C} \in K^{d, \delta} \text { for } \delta<1 \text {. }
$$

Furthermore it will be shown in Sect. 4, Lemma 4.1 that the Schrödinger operator $H-E$ with $H$ given in (1.1) has the strong unique continuation property, so that a solution $\psi \neq \equiv 0$ of $(H-E) \psi=0$ satisfies (1.5). Hence Theorem 1.0 is applicable after some easy coordinate transformations (see below) to the Coulombic case.

Before we state our results for the Coulombic case we reformulate the problem and introduce some notation:

Let $V_{C}$ be given according to (1.2) and suppose that $\psi \not \equiv 0$ satisfies

$$
(H-E) \psi=0 \quad \text { in } B_{\varepsilon}\left(\vec{r}^{\mathrm{CP}}\right)
$$

with $B_{\varepsilon}\left(\vec{r}^{\mathrm{CP}}\right)=\left\{\vec{r} \in \mathbf{R}^{d} \| \vec{r}-\vec{r}^{\mathrm{CP}} \mid<\varepsilon\right\}, \varepsilon>0$, a neighbourhood of the $\mathrm{CP}$, $\vec{r} \mathrm{CP}=\left(\vec{r}_{1}^{\mathrm{CP}}, \vec{r}_{2}^{\mathrm{CP}}, \ldots, \vec{r}_{N}^{\mathrm{CP}}\right)$. Every $\mathrm{CP}$ induces in a natural way a partition of the set of indices of the $N$ particles in $m=m\left(\vec{r}^{\mathrm{CP}}\right)$ clusters $\mathscr{C}_{k}, 1 \leq k \leq m$, where $1 \leq m \leq N-1$, namely: $i, j$ with $1 \leq i, j \leq N$ belong to the same cluster if and only if $\overline{\vec{r}}_{i}^{\mathrm{CP}}=\vec{r}_{j}^{\mathrm{CP}}$. Therefore we can split the potential $V_{C}$ uniquely into the part $U_{C}(\vec{r})$ denoting the interactions between different clusters and the rest describing the interactions in each cluster with more than one element, so

$$
V_{C}=U_{C}\left(\vec{r}_{1}, \ldots, \vec{r}_{N}\right)+\sum_{k,\left|\mathscr{C}_{k}\right| \geq 2} \sum_{\imath<\jmath, i, j \in \mathscr{C}_{k}} \frac{\alpha_{\imath} \alpha_{j}}{\left|\vec{r}_{i}-\vec{r}_{j}\right|} .
$$

Furthermore we note that since $U_{C}\left(\vec{r}_{1}^{\mathrm{CP}}, \ldots, \vec{r}_{N}^{\mathrm{CP}}\right)$ is bounded there is some $B_{R_{0}}\left(\vec{r}^{\mathrm{CP}}\right)$, $R_{0}<\varepsilon$, where $U_{C}$ remains bounded. Now let

$$
x_{\imath}=\sqrt{2 m_{i}}\left(\vec{r}_{i}-\vec{r}_{\imath}^{\mathrm{CP}}\right), \quad 1 \leq i \leq N .
$$

Then (1.9) becomes

$$
\left(-\Delta+V_{\mathrm{CP}}-E\right) \psi_{\mathrm{CP}}=0 \quad \text { in } \quad B_{R}(\mathbf{0}),
$$

where $\psi_{\mathrm{CP}}\left(x_{1}, \ldots, x_{N}\right)=\psi\left(\vec{r}_{1}, \ldots, \vec{r}_{N}\right)$, and

$$
\begin{aligned}
V_{\mathrm{CP}}\left(x_{1}, \ldots, x_{N}\right)= & U_{\mathrm{CP}}\left(x_{1}, \ldots, x_{N}\right) \\
& +\sum_{k,\left|\mathscr{E}_{k}\right| \geq 2} \sum_{i<j, i, j \in \mathscr{E}_{k}} \alpha_{\imath} \alpha_{\jmath}\left|\frac{x_{i}}{\sqrt{2 m_{\imath}}}-\frac{x_{\jmath}}{\sqrt{2 m_{j}}}\right|^{-1} \\
& \text { with } U_{\mathrm{CP}}\left(x_{1}, \ldots, x_{N}\right)=U_{C}\left(\vec{r}_{1}, \ldots, \vec{r}_{N}\right) \\
& \text { and } R \leq \sqrt{2 \min _{1 \leq j \leq N} m_{\jmath}} \varepsilon .
\end{aligned}
$$


So to every fixed coalescence point $\vec{r}^{\mathrm{CP}}$ we associate the coordinate transformation (1.11) and obtain an equation for $\psi_{\mathrm{CP}}$ so that Theorem 1.0 is applicable (once we know that $H-E$ has the strong unique continuation property).

To obtain more detailed and sharper results it will be convenient to use $d$ dimensional polar coordinates $x=r \omega$, where $r=|x|, \omega=x /|x| \in S^{d-1}$ with $S^{d-1}$ the unit sphere in $\mathbf{R}^{d}$.

Let $P_{M}(x)$ be a harmonic homogeneous polynomial of degree $M$ so that $\Delta P_{M}=0$ and $P_{M}(\lambda x)=\lambda^{M} P_{M}(x)$ for $\lambda \in \mathbf{R}$. Then we have (see [18]): $P_{M}(r \omega)=$ $c_{M} r^{M} Y_{M}(\omega)$, where $Y_{M}$ is a real valued normalized surface harmonic, $\int_{S^{d-1}}\left|Y_{M}\right|^{2} d \omega=1$ and $c_{M} \in \mathbf{R}$. The Laplacian in polar coordinates reads

$$
\Delta=\frac{\partial^{2}}{\partial r^{2}}+\frac{d-1}{r} \frac{\partial}{\partial r}-\frac{L^{2}}{r^{2}}
$$

with $-L^{2}$ the Laplace Beltrami operator on $S^{d-1}$. We have

$$
L^{2} Y_{M}=M(M+d-2) Y_{M} .
$$

Equation (1.13) reads now in polar coordinates

$$
\begin{aligned}
V_{\mathrm{CP}}(r \omega)= & U_{\mathrm{CP}}(r \omega)+\frac{1}{r} W_{\mathrm{CP}}(\omega) \\
W_{\mathrm{CP}}(\omega)= & \sum_{k,\left|\mathscr{\zeta}_{k}\right| \geq 2} \sum_{i<j, i, j \in \mathscr{C}_{k}} \alpha_{i} \alpha_{\jmath}\left|\frac{\omega_{\imath}}{\sqrt{2 m_{i}}}-\frac{\omega_{j}}{\sqrt{2 m_{j}}}\right|^{-1} \\
& \text { with } \omega=\left(\omega_{1}, \ldots, \omega_{N}\right)=\left(\frac{x_{1}}{r}, \ldots, \frac{x_{N}}{r}\right) .
\end{aligned}
$$

Our main result about the local behaviour of Coulombic wave functions near a coalescence point can be formulated now.

Theorem 1.1. Let $\psi_{\mathrm{CP}} \not \equiv 0$ be a real valued solution to (1.12) on a neighbourhood of the origin with $V_{\mathrm{CP}}$ given according to (1.14). Let $P_{M}=c_{M} r^{M} Y_{M} \not \equiv 0$ be the harmonic homogeneous polynomial of degree $M$ which determines according to Theorem 1.0 the behaviour of $\psi_{\mathrm{CP}}$ for $r \rightarrow 0$. Then

$$
\psi_{\mathrm{CP}}(r \omega)=P_{M}\left(1+a_{M}+O\left(r^{2}\right)\right)+\eta(r \omega) \quad \text { for } \quad r \rightarrow 0
$$

with

$$
a_{M}=\frac{1}{2 M+d-1} \int_{S^{d-1}} Y_{M}^{2} W_{\mathrm{CP}} d \omega
$$

and

$$
\eta=O\left(r^{M+1}\right), \text { where } \eta \equiv \psi_{\mathrm{CP}}-Y_{M} \int Y_{M}(\omega) \psi_{\mathrm{CP}}(r \omega) d \omega
$$

and $\int_{S^{d-1}} Y_{M} \eta d \omega=0 . W_{\mathrm{CP}}$ is given according to (1.14).

Corollary 1.1. For $\psi_{\mathrm{CP}}$ given by (1.15) let

$$
\psi_{a v}^{(M)}(r)=r^{-M}\left(\int_{S^{d-1}} \psi_{\mathrm{CP}}^{2} d \omega\right)^{1 / 2}
$$


Then

$$
\left(\frac{d}{d r} \psi_{a v}^{(M)}\right)(0)=a_{M} \psi_{a v}^{(M)}(0)
$$

To see this note that by Theorem 1.1

$$
\int_{S^{d-1}} \psi_{\mathrm{CP}}^{2} d \omega=r^{2 M}\left(1+2 a_{M} r+O\left(r^{2}\right)\right) c_{M}^{2}+O\left(r^{2 M+2}\right),
$$

so that $\psi_{a v}^{(M)}(r)=\left(1+a_{M} r+O\left(r^{2}\right)\right)\left|c_{M}\right|$ from which (1.18) immediately follows.

Some remarks might be appropriate:

Remark 1.1. Suppose we consider the Schrödinger equation of a molecule or atom with fixed nuclei. It can be easily seen that the potential can be transformed so that Theorem 1.1 holds. This kind of result was given in [7].

Remark 1.2. Let us point out that the splitting of $\psi_{\mathrm{CP}}$ into $Y_{M} \int_{S^{d-1}} Y_{M} \psi_{\mathrm{CP}} d \omega$ and $\eta$ is natural since $a_{M}$ is uniquely determined by $P_{M}$ whereas $\eta$ is not. This can be seen as follows: Suppose we have another solution $\psi_{1} \neq \psi_{\mathrm{CP}}$ of (1.12) with the following behaviour for $r \rightarrow 0$ : For some $P_{M+1}=c_{M+1} Y_{M+1} r^{M+1}$ we have

$$
\psi_{1}=Y_{M+1} \int_{S^{d-1}} \psi_{1} Y_{M+1} d \omega+\eta_{1}
$$

with $\eta_{1}=O\left(r^{M+2}\right)$ and $Y_{M+1} \int_{S^{d-1}} Y_{M+1} \psi_{1} d \omega=P_{M+1}(1+O(r))$. (In fact it was shown in ([6], Theorem 5.1) that given any $P_{k}$ then a solution satisfying $\psi=P_{k}+o\left(r^{k}\right)$ exists in a neighbourhood of the origin.) Now $\psi_{2}:=\psi_{1}+\psi_{\mathrm{CP}}$ is also a solution to (1.12) and $\psi_{2}=P_{M}\left(1+a_{M} r+O\left(r^{2}\right)\right)+\eta_{2}$, but $\eta_{2}=\eta+P_{M+1}+O\left(r^{M+2}\right)$.

Remark 1.3. If $\vec{r}^{\mathrm{CP}}$ is not a coalescence point, then $V_{\mathrm{CP}}$ is bounded in a neighbourhood of this point and in (1.14) $W_{\mathrm{CP}} \equiv 0$. So Theorem 1.1 remains valid, but with $a_{M}=0$.

Remark 1.4. The nonanalyticity of solutions to Schrödinger equations with Coulombic potentials is already evident for the Hydrogenic case. The first rigorous results about the behaviour of wave functions in the neighbourhood of 2-particle coalescence points date back to the work of Kato [11] in 1957 where for the case that $\psi\left(\vec{r}^{\mathrm{CP}}\right) \neq 0$ the nonanalyticity was analyzed; essentially with a result corresponding to Corollary 1.1 with $M=0$ though with a different averaging. This was generalized for $N$-particle coalescence points in [8] still assuming $\psi(\vec{r} \mathrm{CP}) \neq 0$.

The nonanalyticity of $\psi$ at CP's is also believed to be the main reason for the slow convergence of the usual variational schemes to compute energies of atoms and molecules. There have been successful attempts to incorporate the results of Kato [11] to accelerate the convergence of various numerical schemes for such computations; see e.g. [13].

Another strand to investigate the nonanalyticity of $\psi$ near coalescence points runs under the name Fock-expansions where one tries to find a kind of a generalized power expansion of $\psi_{\mathrm{CP}}$ in terms of powers of $r$ and $\ln r$ with coefficients that are functions in the angles $\omega$. Most of these results concern 2-electron atoms, see [3,1,14, 15] for some recent results. According to these investigations the first nonvanishing term, where logarithms show up is $O\left(r^{M+2}|\ln r|\right.$ ) (in the notation of Theorem 1.1). 
Clearly such a series must have the properties stated in Theorem 1.1 and in particular in Corollary 1.1 so that this first logarithmic term obviously stems from $\eta$.

Remark 1.5. Since the Coulomb potential given in (1.2) depends only on the interparticle distances we immediately see (compare (1.14)) that

$$
W_{\mathrm{CP}}(\omega)=W_{\mathrm{CP}}(-\omega) \text {. }
$$

Without this symmetry property Theorem 1.1 would not hold true. For example take $d=3$ and $V=x_{1}\left(x_{1}^{2}+x_{2}^{2}+x_{3}^{2}\right)^{-1}$, where $\left(x_{1}, x_{2}, x_{3}\right) \in \mathbf{R}^{3}$. Then we can show: Let $\psi$ be a solution of $(-\Delta+V) \psi=0$ in a neighbourhood of the origin, then for some harmonic homogeneous polynomial $P_{M} \not \equiv 0$, we have for $r \rightarrow 0$,

$$
\begin{gathered}
\psi=P_{M}\left(1+a_{M} r+O\left(r^{2}|\ln r|\right)\right)+\eta \\
\text { with } \eta=O\left(r^{M+1}|\ln r|\right) \text { and } \int_{S^{2}} Y_{M} \eta d \omega=0, \\
a_{M}=\frac{1}{2 M+2} \int_{S^{2}}\left(Y_{M}^{2} W\right)(\omega) d \omega \text { with } W(\omega)=r V(r \omega) .
\end{gathered}
$$

To clarify this different kind of local behaviour we shall proceed in the following manner: We shall investigate not directly the Schrödinger equation with the potential $V_{\mathrm{CP}}$ as defined in (1.14) but rather a more general class of potentials and obtain a representation theorem for solutions $u$ of $(-\Delta+V) u=0$, with $V$ belonging to this class. Theorem 1.1 and also (1.20) of the above example will then be special cases.

For this purpose we introduce a Morrey space (see e.g. [4]). Let $\Omega$ be a bounded domain in $\mathbf{R}^{d}$ and $V \in L^{1}(\Omega)$. $V$ is said to belong to $M^{p}(\Omega), 1 \leq p \leq \infty$ if there exists a constant $k$ such that

$$
\int_{\Omega \cap B_{\varrho}}|V| d x \leq k \varrho^{d(1-1 / p)} \text { for all balls } B_{\varrho} .
$$

$B_{\varrho}$ denotes an arbitrary ball of radius $\varrho$ in $\mathbf{R}^{d}$. The norm $\|V\|_{M^{p}(\Omega)}$ is the infimum of the constants $k$ so that $(1.21)$ holds. This and the following proposition can be found in ([4], Lemma 7.18).

Proposition 1.1. Let $\Omega \subset \mathbf{R}^{d}, \Omega$ bounded, $V \in M^{p}(\Omega), 1 / p<\mu$, then

$$
\int_{\Omega}|x-y|^{d(\mu-1)}|V(y)| d y \leq \frac{1-1 / p}{\mu-1 / p}(\operatorname{diam} \Omega)^{d(\mu-1 / p)}\|V\|_{M^{p}} \quad \text { a.e. }(\Omega) \text {. }
$$

Next we set $p=d$ and $\mu=2 / d$. We assume

$$
V \in M^{d}(\Omega), \quad \text { i.e. } \int_{\Omega \cap B_{\varrho}}|V| d x \leq k \varrho^{d-1}
$$

for balls $B_{\varrho}$ of radius $\varrho$ in $\mathbf{R}^{d}$.

Noting that $\forall \Omega^{\prime} \subseteq \Omega,\|V\|_{M^{d}\left(\Omega^{\prime}\right)} \leq\|V\|_{M^{d}(\Omega)}$ we obtain from the above

Proposition 1.2. Let $V \in M^{d}(\Omega), \Omega \subset \mathbf{R}^{d}, \Omega$ bounded, then for $\Omega^{\prime} \subseteq \Omega$

$$
\int_{\Omega^{\prime}}|x-y|^{2-d}|V(y)| d y \leq(d-1)\left(\operatorname{diam} \Omega^{\prime}\right)\|V\|_{M^{d}(\Omega)} \quad \text { a.e. }\left(\Omega^{\prime}\right) .
$$


Remark 1.6. As can be seen from the definition of the Kato class $K^{d},(1.23)$ provides us with an explicit estimate for the integral in (A.1) and will be especially useful for potentials like the Coulombic one (see Proof of Lemma E in Sect. 3).

Before we can state our generalization of Theorem 1.1 we have to collect some basic facts on surface harmonics. Let $\left\{Y_{l, m}\right\}$ be an orthonormal system of real valued surface harmonics of degree $l, l \in \mathbf{N}_{0}$ and $m=0,1, \ldots, h(l)-1$ with

$$
h(l)=\frac{(2 l+d-2)(l+d-3) !}{(d-2) ! l !}
$$

such that

$$
\int_{S^{d-1}} Y_{l, m} Y_{j, k} d \omega=\delta_{l, j} \delta_{m, k}
$$

We note that every $Y_{l, m}$ satisfies

$$
L^{2} Y_{l, m}=l(l+d-2) Y_{l, m},
$$

where $L^{2}$ was defined above. Let $u \in C^{0}\left(B_{R}\right)$ for some $R>0$ and with $B_{R} \subset \mathbf{R}^{d}$ a ball with radius $R$ centered at the origin. We introduce the following orthogonal projections on $S^{d-1}$ :

$$
\left(\mathscr{P}_{l, m} u\right)(r \omega):=Y_{l, m}(\omega) \int_{S^{d-1}} Y_{l, m}(\omega) u(r \omega) d \omega
$$

for $l \in \mathbf{N}_{0}$ and $m=0,1, \ldots, h(l)-1$, and

$$
\begin{aligned}
& \left(\mathscr{P}_{k} u\right)(r \omega)=\sum_{l=0}^{k} \sum_{m=0}^{h(l)-1}\left(\mathscr{P}_{l, m} u\right)(r \omega), \quad k \in \mathbf{N}_{0}, \\
& \left(\mathscr{Q}_{k} u\right)(r \omega)=u(r \omega)-\left(\mathscr{P}_{k} u\right)(r \omega) .
\end{aligned}
$$

Theorem 1.2. Let $B_{\varepsilon}=\left\{x \in \mathbf{R}^{d}:|x|<\varepsilon\right\}$, $\varepsilon>0$, and let $V \in L^{1}\left(B_{\varepsilon}\right)$ be real valued. Assume that $V$ satisfies the assumptions (A.1) and (A.2) with $\Omega=B_{\varepsilon}$ and further that $V$ can be represented as

$$
V(r \omega)=\frac{W(\omega)}{r}+U(r \omega) \text { in } B_{\varepsilon} \text { with } U \text { bounded in } \overline{B_{\varepsilon}} \text { and } W \in L^{2}\left(S^{d-1}\right) \text {. }
$$

Let $u \not \equiv 0$ be a real valued distributional solution of

$$
(-\Delta+V) u=0 \quad \text { in } B_{\varepsilon} .
$$

Then for $r \rightarrow 0$ either $u=O\left(r^{\alpha}\right)$ for all $\alpha>0$ or the behaviour of $u$ near the origin is determined by some harmonic homogeneous polynomial

$$
P_{M}(r \omega)=c_{M} r^{M} Y_{M}, \quad c_{M} \neq 0, \quad M \in \mathbf{N}_{0}, \quad \int_{S^{d-1}} Y_{M}^{2} d \omega=1,
$$

in the following way:

$$
\begin{aligned}
u= & P_{M}\left(1+a_{M} r+\eta_{1}+\eta_{2}\right) \\
& +r^{M+1}\left[\sum_{m=0}^{h(M+1)-1}\left(c_{M} a_{M+1, m} \ln r+\Phi_{M+1, m}\right) Y_{M+1, m}+\eta_{3}\right]
\end{aligned}
$$


with

$$
\begin{aligned}
a_{M} & =\frac{1}{2 M+d-1} \int_{S^{d-1}} W Y_{M}^{2} d \omega, \\
a_{M+1, m} & =\frac{1}{2 M+d} \int_{S^{d-1}} Y_{M+1, m} Y_{M} W d \omega,
\end{aligned}
$$

and where $\eta_{i}=\eta_{i}(r \omega), 1 \leq i \leq 3$, satisfy

$$
\eta_{1}=\left\{\begin{array}{c}
O\left(|\ln r| r^{2}\right) \quad \text { if } \underset{1 \leq m \leq h(M+1)-1}{\max }\left|a_{M+1, m}\right| \neq 0, \\
0 \quad \begin{array}{c}
\text { otherwise } \\
0 \quad
\end{array} \quad \eta_{2}=O\left(r^{2}\right), \\
\Phi_{M+1, m}=O(1) \quad \text { and } \quad \eta_{3}=O(1)
\end{array}\right.
$$

and

$$
\begin{gathered}
\int_{S^{d-1}} \eta_{3}(r \omega) Y_{M}(\omega) d \omega=\int_{S^{d-1}} \eta_{3} Y_{M+1, m} d \omega=0 \\
\text { for } m=0,1, \ldots, h(M+1)-1 .
\end{gathered}
$$

In particular

$$
\left(\int_{S^{d-1}} u^{2} d \omega\right)^{1 / 2}(r)=\left|c_{M}\right| r^{M}\left(1+a_{M} r+\mu_{1}+\mu_{2}\right)
$$

with

$$
\mu_{1}=\left\{\begin{array}{ll}
O\left(\left|r^{2} \ln r\right|^{2}\right) & \text { if } \max _{m}\left|a_{M+1, m}\right| \neq 0, \\
0 & \text { otherwise, }
\end{array} \quad \text { and } \mu_{2}(r)=O\left(r^{2}\right) .\right.
$$

As an immediate consequence we obtain

Corollary 1.2. Suppose that the assumptions of Theorem 1.2 hold and assume that $W(\omega)=W(-\omega)$ for $\omega \in S^{d-1}$. Further let $u$ be a solution of (1.30) which does not vanish faster than polynomially for $r \rightarrow 0$, then for some harmonic homogeneous polynomial $P_{M}$

$$
\begin{gathered}
u(r \omega)=P_{M}\left(1+a_{M} r+O\left(r^{2}\right)\right)+\eta \\
\text { with } \eta=O\left(r^{M+1}\right) \text { and } \int_{S^{d-1}} \eta Y_{M} d \omega=0
\end{gathered}
$$

with $a_{M}$ as above. Furthermore

$$
\left(\int_{S^{d-1}} u^{2} d \omega\right)^{1 / 2}=\left|c_{M}\right| r^{M}\left(1+a_{M} r+O\left(r^{2}\right)\right) .
$$

Remark 1.7. Comparing the results of Theorem 1.2 with the example given in Remark 1.5 [see (1.20)] it now becomes obvious where the logarithms come from. (It is easy to see that the potential in this example satisfies the assumptions of Theorem 1.2.)

We also see that Corollary 1.2 coincides with the results of Theorem 1.1 if we set $u=\psi_{\mathrm{CP}}$ and $W=W_{\mathrm{CP}}$. 
We shall organize the proofs of Theorem 1.1 and 1.2 as follows: In Sect. 2 we shall prove Theorem 1.2 with the help of some lemmas which are proven in Sect. 3. In Sect. 4 we shall show that $V_{\mathrm{CP}}$ satisfies the assumptions of Theorem 1.2 and the strong unique continuation property for solutions to the Coulombic Schrödinger equation, hence proving Theorem 1.1.

\section{Proof of Theorem 1.2}

Suppose $u$ does not vanish faster than polynomially in the origin, then by Theorem 1.0 there is a harmonic homogeneous polynomial $P_{M} \not \equiv 0$ of degree $M \in \mathbf{N}_{0}$ such that

$$
\begin{gathered}
u=P_{M}+\Phi \quad \text { with } \Phi=O\left(r^{M+\nu}\right) \text { for } r \rightarrow 0 \\
\text { with } \nu=\min \left(1, \delta^{\prime}\right), \forall \delta^{\prime} \in[0, \delta) .
\end{gathered}
$$

We denote $P_{M}(r \omega)=c_{M} r^{M} Y_{M}(\omega)$ with $c_{M} \neq 0$ and $Y_{M}$ normalized to 1 .

To verify Theorem 1.2 we proceed similarly as in the proof of Theorem 1.0 (see [6]): We present $u$ by

$$
\begin{aligned}
& u(r \omega)=\sum_{l=0}^{M+1} \sum_{m=0}^{h(l)-1} U_{l, m} Y_{l, m}+Q_{M+1} u, \\
& \text { where } U_{l, m}(r)=\left(\int_{S d-1} u Y_{l, m} d \omega\right)(r)
\end{aligned}
$$

and $Y_{l, m}$ and $Q_{M+1}$ are defined according to (1.25), (1.26) and (1.28), and without loss we take $Y_{M, 0}=Y_{M}$. We shall investigate the behaviour of each term in (2.2) for $r \rightarrow 0$ separately. For the investigation of $U_{l, m}$ we use the following Lemma A (which is an analogue to Lemma $A$ in [6], but yields more information due to the already obtained result (2.1)).

Lemma A. Let $V$ satisfy the assumptions of Theorem 1.2 and let $u$ be a real valued solution of (1.30) with the property (2.1). Then $U_{l, m}, U_{l, m}^{\prime}$ (defined in (2.2), with ' denoting $d / d r)$ are continuous for $r \leq R$ and

$$
u_{l, m}(r):=r^{(d-1) / 2} U_{l, m}(r)
$$

satisfies

$$
-u_{l, m}^{\prime \prime}+\frac{\beta_{l}\left(\beta_{l}-1\right)}{r^{2}} u_{l, m}=F_{l, m} \quad \text { in } \quad(0, R)
$$

in the distributional sense, where $\beta_{l}=l+(d-1) / 2$ and

$$
\begin{aligned}
F_{l, m}(r)= & -\left(\int_{S^{d-1}} Y_{l, m} V u d \omega\right)(r) r^{(d-1) / 2}, \\
& \text { for } m=0,1, \ldots, h(l)-1, \quad \text { and } l=0,1, \ldots, M+1 .
\end{aligned}
$$

Further $U_{l, m}$ has the following behaviour for $r \rightarrow 0$ :

for $l \leq M^{l, m}-1$

$$
U_{l, m}=O\left(r^{M+1}\right), \quad \forall m
$$


for $l=M$

$$
\begin{gathered}
U_{M, m}=O\left(r^{M+1}\right), \quad m=1,2, \ldots, h(M)-1 \\
U_{M} \equiv U_{M, 0}=c_{M} r^{M}\left(1+a_{M} r+\Phi_{M}\right) \quad \text { with } \quad \Phi_{M}=O\left(r^{1+\nu}\right)
\end{gathered}
$$

for $l=M+1$

$$
\begin{aligned}
U_{M+1, m}= & r^{M+1}\left(c_{M} a_{M+1, m} \ln r+\Phi_{M+1, m}\right) \\
& \text { for } \quad m=0,1, \ldots, h(M+1)-1, \quad \text { with } \quad \Phi_{M+1, m}=O(1) .
\end{aligned}
$$

Thereby $a_{M}$ and $a_{M+1, m}$ are defined in (1.33).

Lemma A, which is proven in Sect. 3 directly implies the following

\section{Proposition A.}

$$
u=\mathscr{P}_{M-1} u+\sum_{m=1}^{h(M)-1} \mathscr{P}_{M, m} u+U_{M} Y_{M}+\sum_{m=0}^{h(M+1)-1} \mathscr{P}_{M+1, m} u+\mathscr{Q}_{M+1} u
$$

with

$$
\begin{gathered}
\mathscr{P}_{M-1} u=O\left(r^{M+1}\right), \\
\sum_{m=0}^{h(M)-1} \mathscr{P}_{M, m} u=O\left(r^{M+1}\right), \\
U_{M}(r)=c_{M} r^{M}\left(1+a_{M} r+\Phi_{M}\right) \text { with } \Phi_{M}=O\left(r^{1+\nu}\right),
\end{gathered}
$$

and

$$
\begin{aligned}
& \sum_{m=0}^{h(M+1)-1} \mathscr{P}_{M+1, m} u=r^{M+1} \sum_{m=0}^{h(M+1)-1}\left(c_{M} a_{M+1, m} \ln r+\Phi_{M+1, m}\right) Y_{M+1, m} \\
& \text { with } \quad \Phi_{M+1, m}=O(1), \quad m=0,1, \ldots, h(M+1)-1 .
\end{aligned}
$$

In the second part of the proof of Theorem 1.2 we show

\section{Proposition B.}

$$
\mathscr{Q}_{M+1} u=O\left(r^{M+1}\right)
$$

To verify this we again proceed as in [6]: we first show (Lemma B and C) that $r^{M+1}$ is an upper bound to $\mathcal{Q}_{M+1} u$ in the $L^{2}\left(S^{d-1}\right)$-sense, and then we conclude (Lemma $\mathrm{D}$ and $\mathrm{E}$ ) that $r^{M+1}$ is also a pointwise upper bound to $\mathcal{Q}_{M+1} u$. The lemmas are proven in Sect. 3.

Define

$$
\varphi(r)=\left(\int_{S^{d-1}}\left|\mathcal{Q}_{M+1} u\right|^{2} d \omega\right)^{1 / 2} r^{(d-1) / 2}
$$

then $\varphi$ satisfies a differential inequality given in

Lemma B. Under the assumptions of Lemma $A, Q_{M+1} u$ is continuous in $\overline{B_{R}}$, for $R<$ $\varepsilon,\left|\nabla\left(\mathscr{Q}_{M+1} u\right)\right| \in L^{2}\left(B_{R}\right), \varphi$ is continuous in $[0, R], \varphi(0)=0$ and $\varphi^{\prime} \in L^{2}((0, R), d r)$. 
Furthermore $\varphi$ satisfies for some $\bar{c}>0$,

$$
\begin{gathered}
\int(\chi \varphi)^{\prime} \varphi^{\prime} d r+\beta_{M+1} \beta_{M+2} \int \chi r^{-2} \varphi^{2} d r \leq \bar{c} \int r^{\beta_{M-1}} \varphi \chi d r \quad \text { for all } \quad \chi \geq 0 \\
\chi \in W_{0}^{1,2}(0, R)
\end{gathered}
$$

Therefrom we obtain via a comparison argument

Lemma C. Under the assumptions of Lemma A,

$$
\varphi=O\left(r^{M+1+(d-1) / 2}\right) \text { for } r \rightarrow 0 .
$$

To proceed from this $L^{2}$-bound to a pointwise bound to $\mathscr{Q}_{M+1} u$ we use

Lemma D. Suppose the assumptions of LemmaA hold, then for some $c_{1}(d)$, $c_{2}(d, M)>0$ we have for $|x|=r, r \leq R / 2$,

$$
\begin{aligned}
\left|\left(\mathcal{Q}_{M+1} u\right)(x)\right| \leq & c_{1} r^{-(d+1) / 2} \int_{0}^{2 r} \varphi(t) d t+c_{2} \sup _{|y| \leq 2 r}|u(y)| \sup _{|x|=r} \int_{B_{r}(x)}|x-y|^{2-d} \\
& \times\left(|V(y)|+\int_{S^{d-1}}|V(|y| \omega)| d \omega\right) d y
\end{aligned}
$$

Thereby $B_{r}(x)=\left\{y \in \mathbf{R}^{d}|| x-y \mid<r\right\}$.

We note that for the proof of Lemma D we adapt a subsolution estimate of Hinz and Kalf ([5], Lemma 1) to our problem.

Finally we give upper bounds to the integrals occurring in (2.15):

Lemma E. Under the assumptions of Lemma A we have

$$
\begin{gathered}
\sup _{|x|=r} \int_{B_{r}(x)}\left(|V(y)|+\int_{S^{d-1}}|V(|y| \omega)| d \omega\right)|x-y|^{2-d} d y \leq c(d, R) r \\
\text { for } \quad r \leq R / 2 .
\end{gathered}
$$

Finally combining the foregoing considerations Lemma C, D and E imply

$$
\left|\left(Q_{M+1} u\right)(x)\right| \leq c r^{M+1} \text { for }|x| \leq r \leq R / 2
$$

with $c=c(d, M, R)$, which verifies Proposition $\mathrm{B}$.

The third (and last) part of the proof of Theorem 1.2 will now be established via Proposition A and B: The propositions immediately imply that for $r \rightarrow 0$,

$$
\begin{gathered}
u=U_{M} Y_{M}+\sum_{m=0}^{h(M+1)-1} \mathscr{P}_{M+1, m} u+\mathscr{R} \\
\text { with } \mathscr{R}(r \omega)=O\left(r^{M+1}\right) \quad \text { and } \\
\int_{S^{d-1}} \mathscr{R} Y_{M} d \omega=\int_{S^{d-1}} \mathscr{R} Y_{M+1, m} d \omega=0, \quad m=0,1, \ldots, h(M+1)-1 .
\end{gathered}
$$


Inserting (2.10c) and (2.10d) in (2.18) we obtain

$$
\begin{aligned}
u(r \omega)= & c_{M} r^{M}\left(1+a_{M} r+\Phi_{M}\right) Y_{M} \\
& +r^{M+1} \sum_{m=0}^{h(M+1)-1}\left(c_{M} a_{M+1, m} \ln r+\Phi_{M+1, m}\right) Y_{M+1, m}+\mathscr{B} \\
& \text { with } \Phi_{M+1, m}=O(1) \text { for } m=0,1, \ldots, h(M+1)-1, \\
& \text { and } \Phi_{M}=O\left(r^{1+\min \left(1, \delta^{\prime}\right)}\right) \text { for all } \delta^{\prime} \in[0, \delta) .
\end{aligned}
$$

Now we compare (2.19) with the statements (1.32)-(1.36) in Theorem 1.2. Identifying $\mathscr{R}$ with $\eta_{3} r^{M+1}$ it obviously remains to show that

$$
\Phi_{M}=\eta_{1}+\eta_{2} \quad \text { with } \eta_{1}, \eta_{2} \text { satisfying (1.34) . }
$$

This will finish the proof of the theorem. Clearly (1.37) is just a straightforward consequence of (1.32)-(1.36).

So finally we prove (2.20): Note first that due to (2.8) and (2.19),

$$
u=U_{M} Y_{M}+\mu \text { with } \int_{S^{d-1}} \mu Y_{M} d \omega=0
$$

and

$$
\left(\int_{S^{d-1}} \mu^{2} d \omega\right)^{1 / 2} \leq \mathrm{const} r^{M+1}\left(\sum_{m=0}^{h(M+1)-1}\left|a_{M+1, m}\right||\ln r|+1\right) .
$$

Since $u_{M} \equiv u_{M, 0}$ satisfies (2.4) it is not difficult to see (see also the proof of Lemma A) that

$$
U_{M}(r)=r^{M}\left(c_{M}-J_{M}(r)\right)
$$

where

$$
J_{M}(r)=\int_{0}^{r} t^{-2 \beta_{M}} \int_{0}^{t} F_{M}(s) s^{\beta_{M}} d s d t
$$

with $F_{M} \equiv F_{M, 0}$ defined according to (2.5). Inserting (2.21) into (2.5) we obtain (by taking into account (1.29))

$$
\begin{aligned}
-F_{M}(r) r^{-(d-1) / 2}= & \int_{S^{d-1}} Y_{M} V\left(U_{M} Y_{M}+\mu\right) d \omega \\
= & \int Y_{M}^{2}\left(\frac{W}{r}+U\right) d \omega U_{M}+\int Y_{M} V \mu d \omega \\
= & \left(r^{-1} \int Y_{M}^{2} W d \omega+\int Y_{M}^{2} U d \omega\right) \\
& \times c_{M} r^{M}\left(1+a_{M} r+\Phi_{M}\right)+\int Y_{M} V \mu d \omega,
\end{aligned}
$$


where we made use of (2.8) in the last step. Hence

$$
-F_{M}(r)=2 c_{M} \beta_{M} a_{M} r^{\beta_{M-1}}+r^{\beta_{M}} \eta
$$

where for some constants $C_{\imath}, 1 \leq i \leq 4$,

$$
|\eta| \leq C_{1} \int|U| d \omega+C_{2} r^{-M}\left(\int V^{2} d \omega\right)^{1 / 2}\left(\int \mu^{2} d \omega\right)^{1 / 2}
$$

which by (2.22) gives

$$
|\eta| \leq C_{3}+C_{4} \sum_{m=0}^{h(M+1)-1}\left|a_{M+1, m}\right| \cdot|\ln r|
$$

Combination of (2.26) and (2.24) yields

$$
\begin{aligned}
-J_{M}(r) & =\int_{0}^{r} t^{-2 \beta_{M}} \int_{0}^{t} s^{2 \beta_{M}}\left(2 c_{M} \beta_{M} a_{M} s^{-1}+\eta(s)\right) d s d t \\
& =c_{M} a_{M}+\int_{0}^{r} t^{-2 \beta_{M}} \int_{0}^{t} s^{2 \beta_{M}} \eta(s) d s d t
\end{aligned}
$$

but due to (2.27)

$$
\begin{aligned}
& \int_{0}^{r} t^{-2 \beta_{M}} \int_{0}^{t} s^{2 \beta_{M}}|\eta(s)| d s d t \\
& \quad \leq \int_{0}^{r} t^{-2 \beta_{M}} \int_{0}^{t} s^{2 \beta_{M}}\left(C_{3}+C_{4} \sum_{m=0}^{h(M+1)-1}\left|a_{M+1, m}\right| \cdot|\ln s|\right) d s d t .
\end{aligned}
$$

By partial integration we easily conclude from (2.29),

$$
\int_{0}^{r} t^{-2 \beta_{M}} \int_{0}^{t} s^{2 \beta_{M}}|\eta(s)| d s d t \leq C_{5} \sum_{m=0}^{h(M+1)-1}\left|a_{M+1, m}\right| \cdot|\ln r| r^{2}+C_{6} r^{2}
$$

Combination of (2.23) and (2.28) with inequality (2.30) gives

$$
U_{M}(r)=c_{M} r^{M}\left(1+a_{M} r+\eta_{1}+\eta_{2}\right)
$$


with $\eta_{1}$ and $\eta_{2}$ satisfying (1.34). Since $U_{M}(r)=c_{M} r^{M}\left(1+a_{M} r+\Phi_{M}\right)$ due to (2.10c), this together with the above implies (2.20) finishing the proof of Theorem 1.2.

\section{Proof of the Lemmas}

3.1. Proof of Lemma A. The proof mimicks the proof of Lemma A in [6]. Therefore we give just the main steps.

Let $0<R<\varepsilon$, then due to our assumptions $u$ satisfies

$$
\begin{gathered}
(-\Delta+V) u=0 \text { in } B_{R} \text { in the distributional sense } \\
\text { and } u \in C^{0}\left(\overline{B_{R}}\right) \cap W^{1,2}\left(B_{R}\right) .
\end{gathered}
$$

Define for $l \in \mathbf{N}_{0}$ and $m \in\{0,1, \ldots, h(l)-1\}, \chi(r \omega)=Y_{l, m}(\omega) \eta(r)$, where $\eta \in C_{0}^{\infty}((0, R))$, then

$$
\int u \Delta \chi d x=\int \chi V u d x
$$

Taking into account (1.26) and the definition of $U_{l, m}(r)$ (see (2.2)) resp. $u_{l, m}$ (see (2.3)) we obtain for $\tilde{\eta}=r^{(d-1) / 2} \eta$,

$$
\int u_{l, m}\left(\tilde{\eta}^{\prime \prime}-\frac{\beta_{l}\left(\beta_{l}-1\right)}{r^{2}} \tilde{\eta}\right) d r=-\int \tilde{\eta} F_{l, m} d r
$$

where $F_{l, m}(r)$ (defined in (2.5)) is bounded due to the assumption (1.29) on $V$. Hence (3.3) verifies (2.4).

Now let without loss $\delta<1$ and define

$$
\nu_{\delta}(r):=\int_{0}^{r} t^{1-\delta} \int_{S^{d-1}}|V(t \omega)| d \omega d t
$$

which is finite since

$$
\nu_{\delta}(r) \leq \int_{0}^{r} t^{1-\delta}\left(\int_{S^{d-1}}|W| d \omega t^{-1}+\text { const }\right) d t,
$$

so by $(1.29)$

$$
\nu_{\delta}(r)=O\left(r^{1-\delta}\right)
$$

Therefore

$$
\begin{aligned}
\int_{0}^{t}\left|F_{l, m}(s)\right| d s & =\int_{0}^{t} \int_{S^{d-1}}\left|V Y_{l, m} u\right| d \omega s^{(d-1) / 2} d s \\
& \leq \mathrm{const} \int_{0}^{t}\left(\int_{S^{d-1}}|V| d \omega s^{1-\delta}\right) s^{\beta_{M}-1+\delta} d s \\
& \leq \mathrm{const} t^{\beta_{M}-1+\delta} \nu_{\delta}(t)
\end{aligned}
$$


where we used that $u=O\left(r^{M}\right)$ due to (2.1), and (3.4). Further with

$$
J_{l, m}(r) \equiv \int_{0}^{r} t^{-2 \beta_{l}} \int_{0}^{t} F_{l, m}(s) s^{\beta_{l}} d s d t \quad \text { for } \quad l \leq M
$$

we obtain via (3.5) and (3.6) that

$$
\begin{aligned}
\left|J_{l, m}(r)\right| & \leq \mathrm{const} \int_{0}^{r} t^{M-l-1+\delta} \nu_{\delta}(t) d t \\
& \leq \text { const } \nu_{\delta}(r) \frac{r^{M-l+\delta}}{M-l+\delta} \leq \operatorname{const} r^{M-l+1} \quad \text { for } \quad l \leq M
\end{aligned}
$$

By variation of constants the solution $u_{l, m}$ of (2.4) can be written as

$$
u_{l, m}=r^{\beta_{l}}\left(c_{l, m}-J_{l, m}\right) \quad \text { for } \quad l \leq M
$$

with some constants $c_{l, m} \in \mathbf{R}$.

Further because of (2.2) and the orthonormality of the $Y_{l, m}$ 's we have

$$
\int_{S^{d-1}} u^{2}(r \omega) d \omega=\sum_{l=0}^{M+1} \sum_{m=0}^{h(l)-1} U_{l, m}^{2}(r)+\int\left(\mathscr{Q}_{M+1} u\right)^{2} d \omega
$$

which together with (3.9) and (2.1) immediately implies that $c_{l, m}=0$ for $l \leq M-1$, $m=0,1, \ldots, h(l)-1$ and for $l=M, m=1, \ldots, h(M)-1$. Hence for $r \rightarrow 0$,

$$
U_{l, m}(r)=O\left(r^{M+1}\right) \quad \text { for } \quad \begin{cases}l \leq M-1, & m=0,1, \ldots, h(l)-1 \\ l=M, & m=1, \ldots, h(M)-1\end{cases}
$$

verifying (2.6) and (2.7).

Further by (3.10) and (2.1),

$$
U_{M+1, m}(r)=O\left(r^{M+\nu}\right) \quad \text { for } \quad m=0,1, \ldots, h(M+1)-1,
$$

with $\nu$ given in (2.1) and $\nu=\delta^{\prime}$ since $\delta<1$. Finally from (2.1), (2.2), (2.6), (2.7) and (3.12) we get

$$
Q_{M+1} u=O\left(r^{M+\nu}\right)
$$

To verify (2.8) we give a refined estimate for $F_{M} \equiv F_{M, 0}$ and $J_{M} \equiv J_{M, 0}$ (replacing (3.6) and (3.8)). Note first that due to (2.2), (3.11)-(3.13) $u$ can be written as

$$
u=U_{M} Y_{M}+\mu \text { with } \int \mu Y_{M} d \omega=0 \text { and } \quad \mu=O\left(r^{M+\nu}\right) .
$$


Therefrom we conclude

$$
\begin{aligned}
-F_{M}(r) r^{-(d-1) / 2}= & \int_{S^{d-1}} Y_{M} V\left(U_{M} Y_{M}+\mu\right) d \omega \\
= & \int Y_{M}^{2}\left(\frac{W}{r}+u\right) d \omega U_{M}+\int Y_{M} V \mu d \omega \\
= & U_{M}(r)\left((2 M+d-1) a_{M} r^{-1}\right. \\
& \left.+\int Y_{M}^{2} U d \omega\right)+\int Y_{M}\left(\frac{W}{r}+U\right) \mu d \omega .
\end{aligned}
$$

Since $\left|\int Y_{M} U d \omega\right|=O(1)$ and since by (3.14)

$$
\left|\int Y_{M} V \mu d \omega\right| \leq \mathrm{const}\left(r^{-1} \int|W| d \omega+\int|U| d \omega\right) r^{M+\nu}=O\left(r^{M+\nu-1}\right),
$$

we conclude from the above

$$
-F_{M}(r) r^{-(d-1) / 2}=U_{M}\left((2 M+d-1) a_{M} r^{-1}+O(1)\right)+O\left(r^{M+\nu-1}\right) .
$$

Further since by (2.1)

$$
U_{M}(r)=\int\left(P_{M}+\Phi\right) Y_{M} d \omega=c_{M} r^{M}+O\left(r^{M+\nu}\right)
$$

(3.15) and (3.16) yield

$$
-F_{M}(r)=(2 M+d-1) a_{M} c_{M} r^{\beta_{M-1}}+\eta_{M} \quad \text { with } \quad \eta_{M}=O\left(r^{\beta_{M-1}+\nu}\right) .
$$

Insertion of (3.17) into (3.7) for $l=M, m=0$, gives

$$
\begin{aligned}
J_{M}(r) & =-\int_{0}^{r} t^{-2 \beta_{M}} \int_{0}^{t}\left((2 M+d-1) a_{M} c_{M} s^{\beta_{M-1}}+\eta_{M}(s) s^{\beta_{M}} d s d t\right. \\
& =-a_{M} c_{M} r+O\left(r^{1+\nu}\right) .
\end{aligned}
$$

Application of (3.18) to (3.9) leads to

$$
U_{M}(r)=r^{M}\left(c_{M, 0}+a_{M} c_{M} r+O\left(r^{1+\nu}\right)\right),
$$

and because of (3.16) $c_{M, 0}=c_{M} \neq 0$ verifying (2.8).

Finally we show (2.9): Analogously to (3.9) it is not difficult to see that for $m=0,1, \ldots, h(M+1)-1$,

$$
u_{M+1, m}(r)=r^{\beta_{M+1}}\left(c_{M+1, m}+I_{M+1, m}(r)\right)
$$

with some constants $c_{M+1, m} \in \mathbf{R}$ and with

$$
I_{M+1, m}(r) \equiv \int_{r}^{R} t^{-2 \beta_{M+1}} \int_{0}^{t} F_{M+1, m}(s) s^{\beta_{M+1}} d s d t
$$


Similarly as above we conclude via (3.14) that

$$
\begin{aligned}
-F_{M+1, m}(r) r^{-(d-1) / 2}= & \int_{S^{d-1}} Y_{M+1, m} V\left(U_{M} Y_{M}+\mu\right) d \omega \\
= & U_{M}(r)\left(r^{-1} \int Y_{M} Y_{M+1, m} W d \omega+\int Y_{M} Y_{M+1, m} U d \omega\right) \\
& +\int Y_{M+1, m} V \mu d \omega .
\end{aligned}
$$

But $\int Y_{M} Y_{M+1} U d \omega=O(1)$ and due to (3.14),

$$
\left|\int Y_{M+1, m} V \mu d \omega\right|=O\left(r^{M-1+\nu}\right) \text {, }
$$

therefore

$$
-F_{M+1, m}(r) r^{-(d-1) / 2}=\left((2 M+d) a_{M+1, m} r^{-1}+O(1)\right) U_{M}+O\left(r^{M-1+\nu}\right)
$$

follows and further again by (3.14),

$$
\begin{gathered}
-F_{M+1, m}(r)=(2 M+d) a_{M+1, m} c_{M} r^{\beta_{M-1}}+\eta_{M+1} \\
\text { with } \eta_{M+1}=O\left(r^{\beta_{M-1}+\nu}\right) .
\end{gathered}
$$

Insertion of (3.22) into (3.20) leads to

$$
\begin{aligned}
I_{M+1, m}(r) & =\int_{r}^{R} t^{-2 \beta_{M+1}} \int_{0}^{t} s^{\beta_{M+1}}\left(-(2 M+d) c_{M} a_{M+1, m} s^{\beta_{M-1}}+\eta_{M+1}(s)\right) d s d t \\
& =-a_{M+1, m} c_{M}(\ln R-\ln r)+O(1) \\
& =a_{M+1, m} c_{M} \ln r+O(1) .
\end{aligned}
$$

Finally combining (3.23) with (3.19) we arrive at

$$
u_{M+1, m}(r)=\left(a_{M+1, m} c_{M} \ln r+O(1)\right) r^{\beta_{M+1}},
$$

which verifies (2.9) and finishes the proof of Lemma A.

3.2. Proof of Lemma B. Since the proof of the lemma follows directly the proof of Lemma $B$ in [6] we only indicate the main steps here.

First we recall that $u$ satisfies (3.1). Then due to the assumptions on $V$ we obtain that

$$
Q_{M+1} u \in C^{0}\left(\widetilde{B_{R}}\right) \cap W^{1,2}\left(B_{R}\right) \quad \text { and } \quad \varphi \in C^{0}([0, R]) \cap W^{1,2}((0, R)) .
$$

From now on $Q \equiv Q_{M+1}$. Clearly $\varphi(0)=0$. Then we conclude from (3.1) that

$$
\begin{aligned}
\Delta(Q u)= & Q(V u) \text { in } B_{R} \text { in the distributional sense, } \\
& \text { with } Q(V u) \in L^{1}\left(B_{R}\right),
\end{aligned}
$$

and observe that

$$
Q L^{2} Q \geq(M+2)(M+d) Q
$$


in the quadratic form sense on $L^{2}\left(S^{d-1}\right)$. The above observations lead to (compare [6])

$$
\begin{aligned}
& \int(\chi \varphi)^{\prime} \varphi^{\prime} d r+\beta_{M+1} \beta_{M+2} \int \varphi^{2} r^{-2} \chi d r \leq \int \chi \iint_{S^{d-1}}|\mathcal{Q} u| \cdot|\mathcal{Q}(V u)| d \omega^{d-1} d r \\
& \quad \text { for all } \chi \geq 0, \chi \in W_{0}^{1,2}(0, R)
\end{aligned}
$$

But

$$
\begin{aligned}
& \int \chi \int_{S^{d-1}}|\mathscr{Q} u| \cdot|\mathscr{Q}(V u)| d \omega r^{d-1} d r \\
& \leq \int \chi \int_{S^{d-1}}|\mathcal{Q} u|\left(|V u|+\left|\mathscr{P}_{M+1}(V u)\right|\right) d \omega r^{d-1} d r \\
& \leq \mathrm{const} \int \chi \sup _{|x|=r}|u(x)| \int_{S^{d-1}}|\mathscr{Q} u|\left(|V|+\int_{S^{d-1}}|V| d \omega\right) d \omega r^{d-1} d r \\
& \leq \mathrm{const} \int \chi \sup _{|x|=r}|u(x)| \varphi\left(r^{-1}\left(\int_{S^{d-1}} W^{2} d \omega\right)^{1 / 2}+\left(\int_{S^{d-1}} U^{2} d \omega\right)^{1 / 2}\right) d r \\
& \leq \text { const } \int \chi \varphi r^{\beta_{M-1}} d r
\end{aligned}
$$

where we used $W, U \in L^{2}\left(S^{d-1}\right)$ and (2.1) in the last step. Equation (3.27) together with (3.28) finishes the proof of Lemma B.

3.3. Proof of Lemma $C$. We shall obtain the upper bound to $\varphi$ by a comparison argument: Let

$$
h(r)=\bar{c} r^{\beta_{M+2}}\left(1+\frac{b}{r}\right),
$$

where $\bar{c}$ is the constant occurring on the r.h.s. of the differential inequality (2.13), and $b>0$ is a parameter. We have for $r>0$,

$$
-h^{\prime \prime}+\frac{\beta_{M+1} \beta_{M+2}}{r^{2}} h=\bar{c} b(2 M+d+1) r^{\beta_{M-1}},
$$

so that $h$ satisfies

$$
-h^{\prime \prime}+\frac{\beta_{M+1} \beta_{M+2}}{r^{2}} h \geq \bar{c} r^{\beta_{M-1}} \quad \text { for } \quad r>0 \quad \text { and } \quad b \geq \frac{1}{2 M+d+1} .
$$

Multiplication of this inequality with $\chi \varphi$, where $\chi \geq 0, \chi \in W_{0}^{1,2}(0, R)$ leads to

$$
\int(\chi \varphi)^{\prime} h^{\prime} d r+\int \frac{\beta_{M+1} \beta_{M+2}}{r^{2}} h \chi \varphi d r \geq \bar{c} \int r^{\beta_{M-1}} \chi \varphi d r
$$

which together with inequality (2.13) of Lemma B yields

$$
\int(\chi \varphi)^{\prime}(\varphi-h)^{\prime} d r+\int \frac{\beta_{M+1} \beta_{M+2}}{r^{2}}(\varphi-h) \chi \varphi d r \leq 0 .
$$


Now we choose

$$
\begin{aligned}
& b=\max \left((2 M+d-1)^{-1}, \varphi(R)\left(\bar{c} R^{\beta_{M+1}}\right)^{-1}\right) \\
& \quad \text { so that } \varphi(R) \leq h(R) \text { and (clearly) } \varphi(0)=h(0)=0 .
\end{aligned}
$$

Suppose indirectly that in some interval $\left(a_{1}, a_{2}\right) \subset[0, R], \varphi(r)>h(r)$ and $\varphi\left(a_{i}\right)=h\left(a_{i}\right), i=1,2$. Since $h(r)>0$ in $(0, R]$ and $h$ vanishes polynomially for $r \rightarrow 0$ we can pick $\chi=\eta \varphi^{-1}$ with $\eta \in C_{0}^{\infty}\left(a_{1}, a_{2}\right), \eta \geq 0$ and obtain from (3.33),

$$
\int \eta^{\prime}(\varphi-h)^{\prime} d r+\int \frac{\beta_{M+1} \beta_{M+2}}{r^{2}}(\varphi-h) \eta d r \leq 0 .
$$

Hence $\varphi-h$ satisfies

$$
-(\varphi-h)^{\prime \prime}+\frac{\beta_{M+1} \beta_{M+2}}{r^{2}}(\varphi-h) \leq 0 \quad \text { in } \quad\left(a_{1}, a_{2}\right)
$$

in the distribution sense. But $\varphi-h \in W_{0}^{1,2}\left(\left(a_{1}, a_{2}\right)\right)$, so (3.35) holds also in the weak sense and we arrive at

$$
\int_{a_{1}}^{a_{2}}(\varphi-h)^{\prime 2} d r+\int_{a_{1}}^{a_{2}} \frac{\beta_{M+1} \beta_{M+2}}{r^{2}}(\varphi-h)^{2} d r \leq 0
$$

which is a contradiction. Therefore

$$
\varphi(r) \leq h(r) \text { for } r \leq R,
$$

finishing the proof of Lemma $\mathrm{C}$.

3.4. Proof of Lemma D. This lemma can be considered as the homogeneous version of Lemma D in [6] and its proof remains the same, therefore we indicate only the main steps: Starting with Eq. (3.25) we obtain by Kato's inequality (see $[10,12]$ )

$$
-\Delta|\mathcal{Q} u|-|\mathscr{Q}(V u)| \leq 0 \text { in } B_{R}
$$

in the distribution sense. Mimicking the proof of Lemma 1 in [5] we conclude from (3.38) that for some $c_{1}(d), c_{2}(d)>0$,

$$
|(\mathcal{Q} u)(x)| \leq c_{1} r^{-d} \int_{B_{r}(x)}|(\mathcal{Q} u)(y)| d y+c_{2} \int_{B_{r}(x)}|\mathcal{Q}((V u)(y))| \cdot|x-y|^{2-d} d y
$$

for $x \in B_{R}$ and $r<\operatorname{dist}\left(x, \partial B_{R}\right)$. But for $|x|=r \leq R / 2$ clearly

$$
\begin{aligned}
& \int_{B_{r}(x)}|(Q(V u))(y)| \cdot|x-y|^{2-d} d y \\
& \leq \int_{B_{r}(x)}|x-y|^{2-d}\left(\sum_{l=0}^{M+1} \sum_{m=0}^{h(l)-1}\left|Y_{l, m}\right| \int_{S^{d-1}}\left|Y_{l, m}(V u)(|y| \omega)\right| d \omega+|(V u)(y)|\right) d y \\
& \leq \int_{B_{r}(x)}|x-y|^{2-d}\left(c(M, d) \int_{S^{d-1}}|V(|y| \omega)| d \omega+|V(y)|\right) d y \cdot \sup _{|y| \leq 2 r}|u(y)| \cdot(3.40)
\end{aligned}
$$


Also via Cauchy-Schwarz's inequality

$$
\int_{B_{r}(x)}|(Q u)(y)| d y \leq \int_{0}^{2 r} \int_{S^{d-1}}|(Q u)(t \omega)| d \omega t^{d-1} d t \leq c(d) r^{(d-1) / 2} \int_{0}^{2 r} \varphi(t) d t .
$$

Combining (3.39) with (3.40) and (3.41) we finish the proof of Lemma D.

3.5. Proof of Lemma E. Let $|x|=r \leq R / 2$ and define

$$
J_{r}^{(1)}(x):=\int_{B_{r}(x)}|x-y|^{2-d} \int_{S^{d-1}}|V(|y| \omega)| d \omega d y
$$

then because of assumption (1.29) on $V$ we have

$$
J_{r}^{(1)}(x) \leq K_{1}(d, R) \int_{B_{2 r}(0)}|x-y|^{2-d}|y|^{-1} d y .
$$

It is lengthy but straightforward to work out that

$$
\int_{|y|=t}|x-y|^{2-d} d \omega_{y}=K_{2}(d)(\max (t,|x|))^{2-d}
$$

and therefore for $|x|=r \leq R / 2$,

$$
J_{r}^{(1)}(x) \leq K_{1} K_{2} \int_{0}^{2 r}(\max (t,|x|))^{-d+2} t^{d-2} d t \leq K(d, R) r
$$

where $K_{1}, K_{2}$ and $K$ are some positive constants.

To bound

$$
J_{r}^{(2)}(x):=\int_{B_{r}(x)}|x-y|^{2-d}|V(y)| d y
$$

for $|x|=r \leq R / 2$ from above we use assumption (A.2) on $V$, i.e. $V \in M^{d}\left(B_{\varepsilon}(0)\right)$. Since $B_{r}(x) \subset B_{R}(\mathbf{0}) \subset B_{\varepsilon}(0)$ for $R<\varepsilon, V \in M^{d}\left(B_{r}(x)\right)$, and we obtain via Proposition 1.2

$$
\int_{B_{r}(x)}|x-y|^{2-d}|V(y)| d y \leq(d-1) r\|V\|_{M^{d}\left(B_{R}(\mathbf{0})\right)} \quad \text { for } \quad|x|=r \leq R / 2 .
$$

We note that (3.47) holds pointwise since $V \in K^{d}\left(B_{R}(\mathbf{0})\right)$, which implies that the integral on the 1.h.s. of (3.47) is continuous in $x$ (see [17]). Hence

$$
J_{r}^{(2)}(x) \leq c(d, R) r \quad \text { for } \quad|x|=r \leq R / 2 .
$$

From (3.45) and (3.48) we obtain

$$
J_{r}^{(1)}(x)+J_{r}^{(2)}(x) \leq c(d, R) r \quad \text { for } \quad r \leq R / 2,
$$

finishing the proof of Lemma E. 


\section{Proof of Theorem 1.1}

As already indicated in Sect. 1 the proof of Theorem 1.1 will be organized as follows:

(i) We show that $H-E[H$ given in (1.1) and (1.2)] has the strong unique continuation property. This will imply that $\psi_{\mathrm{CP}}(r \omega)$ vanishes at most polynomially for $r \rightarrow 0$.

(ii) We show that $V \equiv V_{\mathrm{CP}}-E$ satisfies the assumption of Theorem 1.2.

Suppose (i) and (ii) is proven, then we apply Theorem 1.2 to the Schrödinger equation (1.12) and therefore the behaviour of $\psi_{\mathrm{CP}}(r \omega)$ for $r \rightarrow 0$ is characterized by (1.31)-(1.37) with $W(\omega) \equiv W_{\mathrm{CP}}(\omega)$ given according to (1.14). Noting that clearly

$$
\begin{gathered}
W(\omega)=W(-\omega) \text { for } \omega \in S^{d-1} \\
\int_{S^{d-1}} Y_{M+1, m} Y_{M} W d \omega=0 \text { for } m=0,1, \ldots, h(M+1)-1
\end{gathered}
$$

follows due to the symmetry properties of the $Y_{l, m}$ 's. Therefore we obtain (compare (1.33))

$$
a_{M+1, m}=0 \text { for } m=0,1, \ldots, h(M+1)-1 .
$$

Taking this into account in (1.31)-(1.37) the result coincides with the statements (1.15)-(1.17) and finishes the proof of Theorem 1.1.

Hence it remains to show (i) and (ii):

ad $(i)$.

Lemma 4.1. Let $\vec{r}^{(0)}=\left(\vec{r}_{1}^{(0)}, \ldots, r_{N}^{(0)}\right) \in \mathbf{R}^{3 N}$ and $B_{\varepsilon}\left(\vec{r}^{(0)}\right)=\left\{\vec{r} \in \mathbf{R}^{3 N}|| \vec{r}-\vec{r}^{(0)} \mid<\right.$ $\varepsilon\}$. Let $\psi \neq 0, \psi \in C^{0}\left(B_{\varepsilon}\left(\vec{r}^{(0)}\right)\right) \cap W^{1,2}\left(B_{\varepsilon}\left(\vec{r}^{(0)}\right)\right.$ be a real valued distributional solution of

$$
(H-E) \psi=0 \text { in } B_{\varepsilon}\left(\vec{r}^{(0)}\right)
$$

with $H$ denoting the $N$-particle Hamiltonian given in (1.1) and (1.2), and $E \in \mathbf{R}$. If

$$
\int_{B_{\varrho}\left(\vec{r}^{(0)}\right)} \psi^{2}(\vec{r}) d \vec{r}=O\left(\varrho^{\alpha}\right) \text { for all } \alpha>0
$$

then $\psi \equiv 0$ in $B_{\varepsilon}\left(\vec{r}^{(0)}\right)$.

As an immediate consequence we obtain

Corollary 4.1. Let $\psi_{\mathrm{CP}}$ be given according to Theorem 1.1, then if $\psi_{\mathrm{CP}}(r \omega)$ vanishes faster than polynomially for $r \rightarrow 0$, i.e. if (4.5) holds, $\psi_{\mathrm{CP}} \equiv 0$ in $B_{R}(\mathbf{0})$.

Proof of Lemma 4.1. Equation (4.4) explicitly written reads

$$
\left(-\sum_{i=1}^{n} \frac{\Delta_{i}}{2 m_{\imath}}+\sum_{\imath<\jmath}^{n} \frac{\alpha_{i} \alpha_{j}}{\left|\vec{r}_{i}-\vec{r}_{j}\right|}-E\right) \psi=0 \quad \text { for } \quad \vec{r}=\left(\vec{r}_{1}, \ldots, \vec{r}_{N}\right) \in B_{\varepsilon}\left(\vec{r}^{(0)}\right) \text {. }
$$

We reformulate (4.6) by making the following ansatz:

$$
\psi=e^{F} \Phi
$$

with

$$
F\left(\vec{r}_{1}, \ldots, \vec{r}_{N}\right)=\sum_{i<\jmath}^{N} \alpha_{\imath} \alpha_{\jmath} m_{\imath \jmath}\left|\vec{r}_{\imath}-\vec{r}_{\jmath}\right| \quad \text { and } \quad m_{\imath \jmath}=\left(\frac{1}{m_{i}}+\frac{1}{m_{j}}\right)^{-1} .
$$


As will be seen from the following this kind of ansatz is particularly adapted for the Coulomb potential, it was introduced by Leray in [14]. Actually it turns out (by a simple calculation) that since $\Delta_{i}\left|x_{i}-x_{j}\right|=2\left|x_{i}-x_{j}\right|^{-1}$,

$$
\sum_{i=1}^{N} \frac{\Delta_{i}}{2 m_{i}} F=\sum_{i<j}^{N} \frac{\alpha_{i} \alpha_{j}}{\left|\vec{r}_{i}-\vec{r}_{j}\right|}
$$

and

$$
\left|\nabla_{i} F\right| \in L^{\infty}\left(B_{\varepsilon}\left(\vec{r}^{(0)}\right)\right.
$$

By a further calculation we obtain

$$
\begin{aligned}
\sum_{i=1}^{N} \frac{1}{2 m_{i}} \Delta_{i}\left(e^{F} \Phi\right)= & \Phi \sum_{i=1}^{N} \frac{1}{2 m_{i}}\left(\left(\nabla_{i} F\right)^{2}+\Delta_{i} F\right) e^{F} \\
& +2 \sum_{i=1}^{N} \frac{1}{2 m_{i}}\left(\nabla_{i} F \nabla_{i} \Phi\right) e^{F}+e^{F} \sum_{i=1}^{N} \frac{1}{2 m_{i}} \Delta_{i} \Phi
\end{aligned}
$$

and because of (4.6) and (4.9) this yields

$$
\begin{aligned}
& -\sum_{i=1}^{N} \frac{1}{2 m_{i}} \Delta_{i} \Phi-2 \sum_{i=1}^{N} \frac{1}{2 m_{i}}\left(\nabla_{i} F\right)\left(\nabla_{i} \Phi\right) \\
& -\left(\sum_{i=1}^{N} \frac{1}{2 m_{i}}\left(\nabla_{i} F\right)^{2}+E\right) \Phi=0 \\
& \text { with } \sum_{i=1}^{N} \frac{1}{2 m_{i}}\left(\nabla_{i} F\right)^{2}+E \in L^{\infty}\left(B_{\varepsilon}\left(\vec{r}^{(0)}\right)\right) .
\end{aligned}
$$

To Eq. (4.11) we can apply a strong unique continuation result of Hörmander (see [9]) and obtain: If $\Phi$ vanishes faster than polynomially in a point then $\Phi \equiv 0$. But since $e^{F}>0$ and (4.7) holds the same is true for $\psi$ ending the proof of Lemma 4.1. ad (ii).

$V \equiv V_{\mathrm{CP}}-E$ is given according to (1.13) and (1.14). In (1.29) we therefore identify

$$
U(r \omega)=U_{\mathrm{CP}}(r \omega)-E \quad \text { and } \quad W(\omega)=W_{\mathrm{CP}}(\omega)
$$

Due to (1.8) $V$ satisfies assumption (A.1). To verify that $V$ has property (A.2) we use Proposition 4.1. Let $d=3 N, y=\left(y_{1}, \ldots, y_{N}\right), y_{i} \in \mathbf{R}^{3}, 1 \leq i \leq N, B_{\varrho}(\bar{y})=\{y \in$ $\left.\mathbf{R}^{d}|| y-\bar{y} \mid<\varrho\right\}$ with $\varrho>0, \bar{y} \in \mathbf{R}^{3 N}$, then for some $c(d)>0$,

$$
\int_{B_{\varrho}(\bar{y})} \frac{1}{\left|x_{i}-x_{j}\right|} d x \leq c(d) \varrho^{d-1} \quad \forall B_{\varrho}(\bar{y}) \subset \mathbf{R}^{d}, \forall \bar{y} .
$$

Proof of Proposition 4.1. Without loss let $i=1, j=2$. 
Suppose first that $\bar{y}=0$ : Then with $x=\left(x_{1}, \ldots, x_{N}\right)=(r \omega), \omega=\left(\omega_{1}, \ldots, \omega_{N}\right)$, $\omega_{j}=x_{j} / r$,

$$
\begin{gathered}
\int_{B_{\varrho}(\mathbf{0})} \frac{1}{\left|x_{1}-x_{2}\right|} d x=\int_{0}^{\varrho} \int_{S^{d-1}} \frac{1}{r\left|\omega_{1}-\omega_{2}\right|} d \omega r^{n-1} d r=\frac{c_{0}(d)}{d-1} \varrho^{d-1} \\
\text { with } c_{0}(d)=\frac{2 \sqrt{2} \pi^{(3 d-1) / 2}}{\Gamma((3 d-1) / 2)}
\end{gathered}
$$

follows as can be seen from [8].

Now suppose that $\bar{y} \neq 0$ : The case $\bar{y}_{1}=\bar{y}_{2}$ reduces to the foregoing case since by the coordinate transform $y=x-\bar{y}$,

$$
\int_{B_{\varrho}(\bar{y})} \frac{1}{\left|x_{1}-x_{2}\right|} d x=\int_{B_{\varrho}(\mathbf{0})} \frac{1}{\left|y_{1}-y_{2}\right|} d y=\frac{c_{0}(d)}{d-1} \varrho^{n-1} .
$$

Finally let $\bar{y}_{1} \neq \bar{y}_{2}$ : By the transform $y=x-\bar{y}$ we obtain

$$
J_{\varrho} \equiv \int_{B_{\varrho}(\bar{y})} \frac{1}{\left|x_{1}-x_{2}\right|} d x=\int_{B_{\varrho}(\mathbf{0})} \frac{1}{\left|y_{1}-y_{2}+\bar{y}_{1}-\bar{y}_{2}\right|} d y \text {. }
$$

If $\varrho \leq\left|\bar{y}_{1}-\bar{y}_{2}\right| /(2 \sqrt{2})$, then since $\sum_{j=1}^{N} y_{j}^{2}<\varrho^{2}$ we conclude $\frac{1}{\sqrt{2}}\left|y_{1}-y_{2}\right|<\varrho$ and further (because of the assumption on $\varrho$ ) $\left|\bar{y}_{1}-\bar{y}_{2}\right|-\left|y_{1}-y_{2}\right|>\sqrt{2} \varrho$. Hence for some $c^{\prime}(d)<\infty$,

$$
J_{\varrho} \leq \int_{B_{\varrho}(\mathbf{0})} \frac{1}{\left|\bar{y}_{1}-\bar{y}_{2}\right|-\left|y_{1}-y_{2}\right|} d y \leq c_{1}(d) \varrho^{d-1}
$$

If $\varrho>\left|\bar{y}_{1}-\bar{y}_{2}\right| /(2 \sqrt{2})$, then

$$
J_{\varrho} \leq J_{\varrho}^{(1)} J_{\varrho}^{(2)}
$$

with

$$
J_{\varrho}^{(1)}=\int_{\sqrt{y_{1}^{2}+y_{2}^{2}}<\varrho} \frac{d y_{1} d y_{2}}{\left|y_{1}-y_{2}+\bar{y}_{1}-\bar{y}_{2}\right|}
$$

and

$$
J_{\varrho}^{(2)}=\frac{\int}{\sqrt{\sum_{k=3}^{N} y_{k}^{2}<\varrho}} d y_{3} \ldots d y_{n}=c_{2}(d) \varrho^{d-6} .
$$

Further by the transform $z_{1}=\frac{1}{\sqrt{2}}\left(y_{1}-y_{2}\right), z_{2}=\frac{1}{\sqrt{2}}\left(y_{1}+y_{2}\right)$ we obtain with $\bar{z}_{1}=\frac{1}{\sqrt{2}}\left(\bar{y}_{1}-\bar{y}_{2}\right)$

$$
\begin{aligned}
J_{\varrho}^{(1)} & =\int_{\sqrt{z_{1}^{2}+z_{2}^{2}<\varrho}} \frac{d z_{1} d z_{2}}{\left|z_{1}+z_{2}\right|} \leq \int_{\left|z_{1}\right|<\varrho} \frac{d z_{1}}{\left|z_{1}+\bar{z}_{1}\right|} \int_{\left|z_{2}\right|<\varrho} d z_{2} \\
& =\int_{\left|z-\bar{z}_{1}\right|<\varrho} \frac{d z}{|z|} c_{3}(d) \varrho^{3} \leq \int_{|z|<4 \varrho} \frac{d z}{|z|} c_{4}(d) \varrho^{3}=c_{4}(d) \varrho^{5},
\end{aligned}
$$


where in the last step we used that due to the assumption on $\varrho,\left|\bar{z}_{1}\right|<2 \varrho$.

Combining (4.18)-(4.20)

$$
J_{\varrho} \leq c(d) \varrho^{d-1}
$$

follows. This proves Proposition 4.1.

Proposition 4.1 clearly implies that $V_{C}$ given in (1.2) satisfies assumption (A.2) for all $\Omega \subset \mathbf{R}^{d}$. Since $V_{\mathrm{CP}}\left(x_{1}, \ldots, x_{N}\right)=V_{C}\left(\vec{r}_{1}, \ldots, \vec{r}_{N}\right)$ with $x_{i}=\sqrt{2 m_{i}}\left(\vec{r}_{i}-\vec{r}_{i}^{(\mathrm{CP})}\right)$, $1 \leq i \leq N$ it follows immediately that $V_{\mathrm{CP}}$ satisfies also (A.2), and further since $E=$ const, $V=V_{\mathrm{CP}}-E$ has this property.

Further we note that due to the definition of $U_{\mathrm{CP}}, U_{\mathrm{CP}}$ is bounded, and finally we observe that $W_{\mathrm{CP}} \in L^{2}\left(S^{d-1}\right)$ since $\left|x_{i}-x_{j}\right|^{-1} \in L_{\mathrm{loc}}^{2}\left(\mathbf{R}^{3 N}\right)$ and $r^{-1} \in$ $L^{2}\left([0, R], r^{d-1} d r\right)$. Hence $V=V_{\mathrm{CP}}-E$ satisfies the assumption necessary to apply Theorem 1.2.

\section{Fermionic Wave Functions}

In this final section we investigate the influence of the Pauli principle on the local behavior of fermionic wave functions. Let

$$
H \psi=E \psi \quad \text { in } \quad \Omega \in \mathbf{R}^{3 N^{\prime}}
$$

where

$$
H=-\sum_{i=1}^{N^{\prime}} \frac{\Delta_{\imath}}{2 m_{\imath}}+V\left(\vec{r}_{1}, \ldots, \vec{r}_{N^{\prime}}\right),
$$

$\vec{r}_{i} \in \mathbf{R}^{3}, m_{i}>0$ for $i=1,2, \ldots, N^{\prime}, E \in \mathbf{R}$, and

$$
V=\sum_{\imath<j}^{N^{\prime}} V_{i \jmath}\left(\left|\vec{r}_{\imath}-\vec{r}_{\jmath}\right|\right)
$$

where $V$ satisfies the assumptions of Theorem 1.0, so $d=3 N^{\prime}$. The Hamiltonian $H$ in (5.1) describes a $N^{\prime}$-particle system and in particular with $V=V_{C}$ as in (1.2) atoms and molecules. Now suppose $N \leq N^{\prime}$ particles are fermions of the same kind e.g., electrons and without loss we denote the coordinates of these particles by $\left(\vec{r}_{1}, \ldots, \vec{r}_{N}\right)$. Then we have $m_{\imath}=m$ for some $m>0$ for $1 \leq i \leq N$ and

$$
\begin{gathered}
V_{\imath j}(r)=V_{12}(r), \quad \forall r>0, \quad \text { with } 1 \leq i<j \leq N, \\
\text { and } \quad V_{1 k}(r)=V_{j k}(r), \quad \forall r>0 \quad \text { with } 2 \leq j \leq N, N<k \leq N^{\prime} .
\end{gathered}
$$

Then the wave function has to transform with respect to these coordinates according to a specific representation of $\mathscr{S}_{N}$ [10]. This property will imply that such a wave function has to vanish at least of some order (depending on $N$ ) at coalescence points $\vec{r}^{\mathrm{CP}}$, where the coordinates of the fermions coincide. We make a coordinate transformation as in (1.11) (with $N$ replaced by $N^{\prime}$ ) so that (5.1) becomes

$$
\left(-\Delta+V_{\mathrm{CP}}-E\right) \psi_{\mathrm{CP}}=0 \quad \text { in } \quad B_{R}(\mathbf{0}) \text {. }
$$

If $\psi_{\mathrm{CP}}$ does not vanish faster than polynomially in $\mathbf{0}$ than by Theorem 1.0 we have for some homogeneous harmonic polynomial $P_{M} \neq 0$,

$$
\begin{aligned}
& \psi_{\mathrm{CP}}\left(x_{1}, \ldots, x_{N}, x_{N+1}, \ldots, x_{N^{\prime}}\right) \\
& \quad=P_{M}\left(x_{1}, \ldots, x_{N}, x_{N+1}, \ldots, x_{N^{\prime}}\right)+o\left(|x|^{M}\right) .
\end{aligned}
$$


We first consider the case that $\psi$ is totally antisymmetric with respect to $\vec{r}_{1}, \ldots, \vec{r}_{N}$ (this corresponds to the case that the total spin of the $N$ electrons is $s=N / 2$ ). But due to the foregoing coordinate-transformation this implies also that $\psi_{\mathrm{CP}}$ is totally antisymmetric with respect to $x_{1}, \ldots, x_{N}$, i.e.

$$
\begin{aligned}
& \psi_{\mathrm{CP}}\left(x_{1}, \ldots, x_{i}, \ldots, x_{\jmath}, \ldots, x_{N}, x_{N+1}, \ldots, x_{N^{\prime}}\right) \\
& \quad=-\psi_{\mathrm{CP}}\left(x_{1}, \ldots, x_{j}, \ldots, x_{\imath}, \ldots, x_{N}, x_{N+1}, \ldots, x_{N^{\prime}}\right) \\
& \quad \text { for } \quad 1 \leq i<j \leq N .
\end{aligned}
$$

But because of (5.4) this implies that

$$
\begin{aligned}
& P_{M}\left(x_{1}, \ldots, x_{i}, \ldots, x_{\jmath}, \ldots, x_{N}, x_{N+1}, \ldots, x_{N^{\prime}}\right) \\
& \quad+P_{M}\left(x_{1}, \ldots, x_{\jmath}, \ldots, x_{\imath}, \ldots, x_{N}, x_{N+1}, \ldots, x_{N^{\prime}}\right)=o\left(|x|^{M}\right) \\
& \text { for } \quad|x| \rightarrow 0 \text { for } 1 \leq i<j \leq N .
\end{aligned}
$$

Hence $P_{M}$ is totally antisymmetric with respect to $x_{1}, \ldots, x_{N}$.

Remark 5.1. Other symmetry properties of $\psi_{\mathrm{CP}}$ (e.g. angular momentum and parity) will carry over to $P_{M}$ according to (5.4) analogously.

Therefore to determine the minimal order of vanishing of $\psi_{\mathrm{CP}}$ in $\mathbf{0}$ it suffices to determine the minimal degree of $P_{M} \neq \equiv 0$, and obviously it suffices to consider homogeneous harmonic polynomials which do not depend on the coordinates $x_{N+1}, \ldots, x_{N^{\prime}}$.

Lemma 5.1. Let $P_{M}\left(x_{1}, \ldots, x_{N}\right) \not \equiv 0$ be a totally antisymmetric harmonic homogeneous polynomial with degree $M$. Then $M$ is greater than or equal to the degree of the totally antisymmetric harmonic homogeneous polynomial

$$
D^{(N)}\left(x_{1}, \ldots, x_{N}\right)=\frac{1}{\sqrt{N !}} \sum_{\sigma \in \mathscr{K} N} \operatorname{sgn} \sigma \prod_{i=1}^{N} \varphi_{i}\left(x_{\sigma(i)}\right) .
$$

Thereby the functions $\varphi_{\imath}=\varphi_{i}(y), y=\left(y_{1}, y_{2}, y_{3}\right) \in \mathbf{R}^{3}$ are monomials ordered such that their degree is monotonically increasing:

$$
\begin{gathered}
\varphi_{1}(y)=1, \varphi_{2}(y)=y_{1}, \varphi_{3}(y)=y_{2}, \varphi_{4}(y)=y_{3}, \\
\varphi_{5}(y)=y_{1}^{2}, \varphi_{6}(y)=y_{1} y_{2}, \ldots, \varphi_{10}(y)=y_{3}^{2}, \\
\varphi_{11}(y)=y_{1}^{3}, \varphi_{12}(y)=y_{1}^{2} y_{2}, \ldots
\end{gathered}
$$

Remark 5.2. $D^{(N)}\left(x_{1}, \ldots, x_{N}\right)$ in (5.7) is the well known Slater determinant of $\varphi_{1}, \ldots, \varphi_{N}$. Note that the ordering of the $\varphi_{i}$ is of course not unique but this is irrelevant for our considerations.

Proof of Lemma 5.1. Clearly $D^{(N)}\left(x_{1}, \ldots, x_{N}\right)$ is a totally antisymmetric homogeneous polynomial. To see that it is harmonic note that

$$
\begin{aligned}
\Delta D^{(N)}\left(x_{1}, \ldots, x_{N}\right) & =\Delta \frac{1}{\sqrt{N !}} \sum_{\sigma \in \mathscr{Y}_{N}} \operatorname{sgn} \sigma \prod_{i=1}^{N} \varphi_{\sigma(i)}\left(x_{\imath}\right) \\
& =\frac{1}{\sqrt{N !}} \sum_{j=1}^{N} \sum_{\sigma \in \mathscr{K}_{N}} \operatorname{sgn} \sigma \Delta_{j} \varphi_{\sigma(j)}\left(x_{j}\right) \prod_{i \neq j}^{N} \varphi_{\sigma(i)}\left(x_{\imath}\right) .
\end{aligned}
$$

But for each $j, \Delta_{\jmath} \varphi_{\sigma(\jmath)}\left(x_{\jmath}\right)$ is a linear combination of monomials in $x_{j}$ which already occur amongst the other monomials of lower degree, hence $\Delta D^{(N)}\left(x_{1}, \ldots, x_{N}\right)=0$. 
Let $g(N)$ denote the degree of $D^{(N)}$, then clearly $g(N)$ is equal the degree of $\prod_{i=1}^{N} \varphi_{\imath}\left(x_{\imath}\right)$. Now let $P_{M}$ be given according to our assumptions, then we have to verify that $M \geq g(N) . P_{M}$ can be represented as a finite sum of monomials $q_{i}\left(x_{1}, \ldots, x_{N}\right)$ of degree $M$ and since $P_{M}$ is antisymmetric it follows that

$$
q_{i}\left(x_{1}, \ldots, x_{N}\right)=\prod_{j=1}^{N} q_{i j}\left(x_{j}\right)
$$

with the $q_{i j}$ being monomials in $x_{j}$, where for each $i$ at most one of the $q_{i j}$ equals a constant. Comparison with our construction (5.8) clearly implies that the degree of $q_{i} \geq g(N)$, verifying our lemma.

Denote by $n_{m}$ the cardinality of the subset of monomials $\varphi_{i}, 1 \leq i \leq N$ as given in (5.8), which have a given degree $m$; then clearly

$$
n_{m}=\frac{1}{2}(m+1)(m+2) \text {. }
$$

Define

$$
k(N)=\max \left\{k \in \mathbf{N}_{\mathbf{0}} \mid \sum_{m=0}^{k} n_{m} \leq N\right\}
$$

and let

$$
b(N)=N-\sum_{m=0}^{k(N)} n_{m} .
$$

Suppose $P_{M}$ has minimal degree, then if $b(N)=0, P_{M}$ clearly is uniquely determined (up to a multiplicative constant), whereas otherwise there are $\left(\begin{array}{c}n_{k+1} \\ b\end{array}\right)$ such polynomials.

Theorem 5.1. Let $P_{M}\left(x_{1}, \ldots, x_{N}\right) \not \equiv 0$ be a totally antisymmetric harmonic homogeneous polynomial of degree $M(N)$, then

$$
M(N) \geq g(N) \quad \text { where } g(N)=\frac{1}{8} k(k+1)(k+2)(k+3)+b(N)(k+1)
$$

with $k(N)=[\alpha(N)]$, the integer part of $\alpha(N)$, and $\alpha(N)$ is the unique positive root of the cubic equation

$$
(\alpha+1)(\alpha+2)(\alpha+3)=6 N, \quad \text { and } \quad b(N)=N-\frac{1}{6}(k+1)(k+2)(k+3) .
$$

Proof of Theorem 5.1. With $g(N)$ denoting the degree of $D^{(N)}\left(x_{1}, \ldots, x_{N}\right)$ as given in (5.7), Lemma 5.1 implies that (5.12) holds. Further with $k(N)$ and $b(N)$ given according to (5.10) and (5.11), we obtain

$$
g(N)=\sum_{m=0}^{k(N)} n_{m} m+b(N)(k+1) .
$$

But,

$$
\begin{gathered}
\sum_{m=0}^{k(N)} n_{m} m=\frac{1}{8} k(k+1)(k+2)(k+3) \\
\sum_{m=0}^{k(N)} n_{m}=\frac{1}{6}(k+1)(k+2)(k+3) .
\end{gathered}
$$


Therefore, by (5.10) we conclude $k(N)=[\alpha(N)]$ with $\alpha$ given as above. This proves Theorem 5.1.

To make the foregoing results more transparent particularly with respect to large $N$-behavior we replace $g(N)$ by $g(x)$ for the real variable $x \geq 1$ and derive upper and lower bounds $B_{ \pm}$to $g$ which have the same leading order.

Let $g(1)=0$ and let $g(N)$ be given according to (5.12) for $N \geq 2$. We define

$$
g(x)=g(N)+(g(N+1)-g(N))(x-N) \quad \text { for } \quad x \in[N, N+1], \quad \forall N \in \mathbf{N} .
$$

Then due to (5.13) the slope of the line segment between $(N, g(N))$ and $(N+1, g(N+$ $1)$ ) is given by $g(N+1)-g(N)=k(N)+1$. Next we define a sequence of integers $\left\{N_{l}\right\}, l \in \mathbf{N}_{0}$ recursively:

$$
\begin{aligned}
N_{0} & =1, \\
N_{1} & =\min \left\{N \in \mathbf{N} \mid k(N)=k\left(N_{0}\right)+1\right\}=4, \\
N_{l+1} & =\min \left\{N \in \mathbf{N} \mid k(N)=k\left(N_{l}\right)+1\right\} .
\end{aligned}
$$

Therefore, recalling the definition of $k(N)$, Eq. (5.10),

$$
k\left(N_{l+1}\right)=k\left(N_{l}\right)+1=l+1, \quad \forall l \in \mathbf{N}_{0} .
$$

From the above we conclude that $\{(x, g(x)) \mid x \geq 1\}$ is a polygonal path joining the points $\left(N_{l}, g\left(N_{l}\right)\right)$ by straight lines with slope $k\left(N_{l+1}\right)$. Also, it is easy to check that our construction implies for all $x, y \geq 2$,

$$
g(x+y) \geq g(x)+g(y) \geq 2 g\left(\frac{x+y}{2}\right) .
$$

Corollary 5.1. Let $g(x)$ be given according to (5.16). Then,

$$
B_{-}(x) \leq g(x) \leq B_{+}(x) \text { for } x \geq 1,
$$

where

$$
\begin{aligned}
& B_{-}(x)=\frac{3}{4} x \alpha(x), \\
& B_{+}(x)=\frac{3}{4} x \beta(x)+\frac{5}{96}(\beta(x)+2)^{2}-\frac{3}{128}
\end{aligned}
$$

with $\alpha(x)$ given according to (5.13) (with $N$ replaced by $x$ ), and $\beta(x)$ is the unique positive root of the cubic equation

$$
(\beta+1)(\beta+2)(\beta+3)-\frac{1}{4}(\beta+2)=6 x .
$$

Proof of Corollary 5.1. Note first that it is not difficult but lengthy to verify that both $B_{+}$are convex functions in $x$ (this can be done using the chain rule for $B_{-}(x(\alpha)$ ) with $x(\alpha)$ being the inverse function to $\alpha(x)$, and analogously for $B_{+}(x(\beta))$. Further, due to the foregoing considerations [see Eq. (5.19)], also $g(x)$ is convex. Now we take into account that obviously

$$
\alpha\left(N_{l}\right)=k\left(N_{l}\right) \quad \text { and } \quad b\left(N_{l}\right)=0 \quad \forall l \in \mathbf{N}_{0},
$$

so that due to (5.13),

$$
B_{-}\left(N_{l}\right)=\frac{3}{4} N_{l} k=\frac{1}{8} k(k+1)(k+2)(k+3)=g\left(N_{l}\right) \quad \text { with } \quad k=k\left(N_{l}\right) .
$$

The above implies that the line segments of $g$ are secants to $B_{-}$and therefore $g(x) \geq B_{-}(x)$ for all $x \geq 1$. To verify that also $g(x) \leq B_{+}(x)$ for $x \geq 1$ we 
show that there exists a monotonically increasing sequence $\left\{x_{l}\right\}$ with $x_{l} \in\left(N_{l}, N_{l+1}\right)$ such that

$$
B_{+}\left(x_{l}\right)=g\left(x_{l}\right)
$$

and

$$
\frac{d B_{+}}{d x}\left(x_{l}\right)=\frac{d g}{d x}\left(x_{l}\right)=l+1,
$$

which implies that $B_{+}$is the envelope of the line segments of $g$. To show this, we define for $l \in \mathbf{N}_{0}, \beta_{l}=l+\frac{1}{2}$ and [compare (5.21)]

$$
x_{l}=\frac{1}{6}\left(\beta_{l}+2\right)\left(\left(\beta_{l}+1\right)\left(\beta_{l}+3\right)-\frac{1}{4}\right) \text {. }
$$

Then,

$$
x_{l}=\frac{1}{12}(2 l+5)\left(l^{2}+5 l+5\right) .
$$

Obviously, $x_{l} \notin \mathbf{N}_{0}$, and due to (5.21), $\beta_{l}=\beta\left(x_{l}\right)$.

Now we show that $x_{l} \in\left(N_{l}, N_{l+1}\right)$ : Let $k\left(x_{l}\right) \equiv\left[\alpha\left(x_{l}\right)\right]$ with $\alpha$ satisfying

$$
(\alpha+1)(\alpha+2)(\alpha+3)=6 x_{l} .
$$

With $k=k\left(x_{l}\right)$ we obtain via (5.26)

$$
(k+1)(k+2)(k+3) \leq(l+5 / 2)\left(l^{2}+5 l+5\right) \leq(k+2)(k+3)(k+4),
$$

and this implies

$$
\left[\alpha\left(x_{l}\right)\right]=l \quad \forall l \in \mathbf{N}_{0} .
$$

Since $\alpha(x)$ is monotonically increasing, and due to (5.17) and (5.22) $\alpha\left(N_{l}\right)=l$ we obtain $x_{l} \in\left(N_{l}, N_{l+1}\right)$.

Calculating now $g\left(x_{l}\right)$ and $B_{+}\left(x_{l}\right)$ we arrive at

$$
B_{+}\left(x_{l}\right)=\frac{1}{24}(l+1)^{2}\left(3 l^{2}+18 l+26\right)=g\left(x_{l}\right),
$$

verifying (5.24). Furthermore, we find by a straightforward but lengthy calculation

$$
\frac{d B_{+}}{d x}\left(x_{l}\right)=\left(\frac{d B_{+}(x(\beta))}{d \beta} \cdot\left(\frac{d x(\beta)}{d \beta}\right)^{-1}\right)_{\beta=l+1 / 2}=l+1,
$$

verifying (5.25) and finishing the proof of Corollary 5.1 .

Remark 5.3. Obviously, $B_{ \pm}(N)$ can both be given explicit by using Cardano's formula for cubic equations, but to make their asymptotic behavior, and hence also the behavior of $g(N)$ for large $N$ more transparent, we expand these bounds in powers of $N$, estimate the remainder and get

$$
\begin{gathered}
\frac{1}{24}(6 N)^{2 / 3}-\frac{1}{648}(6 N)^{-2 / 3}<g(N)-\frac{1}{8}(6 N)^{4 / 3} \\
+\frac{3}{2} N<\frac{5}{48}(6 N)^{2 / 3}+\frac{23}{1152}+\frac{125}{20736}(6 N)^{-2 / 3} .
\end{gathered}
$$

It is now easy to generalize Theorem 5.1 for general spin states, taking into account well known properties of the representation theory of the symmetric group $\mathscr{S}_{N}$ [10].

Theorem 5.2. Let $\psi_{\mathrm{CP}}\left(x_{1}, \ldots, x_{N}, x_{N+1}, \ldots, x_{N^{\prime}}\right)$ be given according to (5.3) and (5.4) and let $N_{1} \geq N_{2} \geq 0$ such that $N_{1}+N_{2}=N$. Suppose that $\psi_{\mathrm{CP}}$ is totally antisymmetric with respect to the coordinates $x_{1}, \ldots, x_{N_{1}}$ and to the coordinates 
$x_{N_{1}+1}, \ldots, x_{N_{1}+N_{2}}$. Then the order of vanishing of $\psi_{\mathrm{CP}}$ in the $N$-fermionic coalescence point $\mathbf{0}$ - which equals the degree $M$ of the polynomial $P_{M}$ - is at least

$$
M\left(N_{1}, N_{2}\right)=g\left(N_{1}\right)+g\left(N_{2}\right) .
$$

Furthermore, for fixed $N$ the minimum value of the degree is given by

$$
M_{0}(N):=\min _{N_{1}+N_{2}=N} M\left(N_{1}, N_{2}\right)= \begin{cases}2 g\left(\frac{N}{2}\right), & N \text { even } \\ g\left(\frac{N+1}{2}\right)+g\left(\frac{N-1}{2}\right), & N \text { odd } .\end{cases}
$$

Proof of Theorem 5.2. Analogously to the foregoing considerations (compare (5.4), (5.5), (5.6)) the polynomial $P_{M}$ is totally antisymmetric with respect to the coordinates $x_{1}, \ldots, x_{N_{1}}$ and to the coordinates $x_{N_{1}+1}, \ldots, x_{N_{1}+N_{2}}$. According to Lemma 5.1 such a polynomial with minimal degree can be constructed as a product of two Slaterdeterminants of degrees $g\left(N_{1}\right)$ and $g\left(N_{2}\right)$. This yields (5.29), whereas (5.30) is an immediate consequence of the convexity of $g(x)$ (compare (5.19)).

We note that the setting of Theorem 5.2 corresponds to a wave function of a system with $N=N_{1}+N_{2}$ identical fermions whose total spin is $s=\frac{1}{2}\left(N_{1}-N_{2}\right)$. The Pauli principle requires the wave function to be antisymmetric in $N_{1}$ and antisymmetric in the other $N_{2}$ fermions. In order to be an irreducible representation of $\mathscr{S}_{N}$ also symmetrisation in the way how to pick up any $N_{2}$ fermions out of $N$ is necessary. This, however, makes every polynomial $P_{M}$ with minimal degree a superposition of products of two determinants, hence it does not change anything on the degree $M\left(N_{1}, N_{2}\right)$.

Remarks (5.4). i) As numerical example we compute that for $N=1000, M_{0}(1000)$ is given by $2 g(500)=9360$, whereas $g(1000)=12155$. However, (5.28) yields, rewritten for $g(500)$ resp. $g(1000)$,

$$
\begin{aligned}
& 4667.103<g(500)<4680.1235 \\
& 12142.16<g(1000)<12162.82 .
\end{aligned}
$$

Similarly, for $N=20, M_{0}(20)=2 g(10)=30, g(20)=45$ we find

$$
\begin{aligned}
& 15-\left(2.19 \cdot 10^{-6}\right)<g(10)<15.98 \\
& 45-\left(8.69 \cdot 10^{-7}\right)<g(20)<46.54
\end{aligned}
$$

ii) The behaviour of $g(N) \sim N^{4 / 3}$ reminds oneself on Thomas-Fermi theory although no energy consideration has been made.

iii) Note that for atoms $M_{0}(N)$ is a lower bound to the order of vanishing of a manyfermion wave function at $N$-fermion CPs. Certainly it is not always attained - atoms with ground states with total spin $>1 / 2$ exist, but we believe that it is of the right order of magnitude for $N \rightarrow \infty$, though we have no clue how to prove this. For other systems our lower bound is attained: Take an isotropic $N$-particle harmonic oscillator with Hamiltonian

$$
H=\sum_{i=1}^{N}\left(-\Delta_{i}+x_{i}^{2}\right),
$$


then with $P_{M}$ being any harmonic homogeneous polynomial in $3 N$ variables, $u=e^{-|x|^{2} / 2} P_{M}$, is an eigenfunction so that

$$
H u=(2 M+3 N) u .
$$

iv) In our construction of a "minimal" polynomial $D^{(N)}\left(x_{1}, \ldots, x_{N}\right)$ we have chosen the $N$-fermion $\mathrm{CP}, x_{\mathrm{CP}}$, to be the origin. It is easy to see that (5.7) is translationally invariant, i.e.

$$
D^{(N)}\left(x_{1}+\vec{a}, x_{2}+\vec{a}, \ldots, x_{N}+\vec{a}\right)=D^{(N)}\left(x_{1}, x_{2}, \ldots, x_{N}\right) .
$$

This follows immediately from the construction of $D^{(N)}$, since adding a constant vector to any coordinate $x_{i}$ changes the rows of the determinant (5.7) by adding a multiple of another row, which leaves the determinant invariant. Furthermore, $D^{(N)}$ also reproduces the correct behavior of wave functions at $N-1$ fermion CP's if one changes one coordinate, e.g. $x_{N}$, to $x_{N}+\vec{a}$. This can be easily checked since then $D^{(N)}$ contains either all the monomials $\left(x_{N}+a\right)_{i}^{k(N)}, i=1,2,3$, or one or two of the monomials $\left(x_{N}+a\right)_{\imath}^{k(N)+1}$, depending whether $N$ is one of the $N_{l}$ in (5.17) or not. The minor of these elements is given exactly by one of the equivalent $D^{(N-1)}\left(x_{1}, \ldots, x_{N-1}\right)$, therefore the lower bound to the order of vanishing of the wave function is $g(N-1)$ as required. Analogously, one can easily conclude from the behavior of $D^{(N)}$ at $(N-n)$-fermion CP's the lower bound to the order of vanishing of the wave function to be $g(N-n)$.

Finally, let us note we can easily generalize our results for antisymmetric wave functions for a system of $N$ identical particles living in $\nu$ space dimensions. Since our Theorem 1.0 for $N^{\prime}$-particle wave functions holds also for systems of $N^{\prime}$ identical $\nu$-dimensional particles (under suitable assumptions on the two body potentials), we can generalize (5.1) and (5.2) to this case. Suppose the wave function $\psi\left(\vec{r}_{1}, \ldots, \vec{r}_{N^{\prime}}\right)$, where the $\vec{r}_{i} \in \mathbf{R}^{\nu}$ denote the $\nu$-dimensional position vectors, is totally antisymmetric with respect to $N \leq N^{\prime}$ particles, then it must vanish again for $N$-particle CP's at least like $g_{\nu}(N)$ given by

$$
g_{\nu}(N)=\frac{\nu(k+\nu) !}{(k-1) !(\nu+1) !}+b_{\nu}(N)(k+1),
$$

where $k=k(N)=\left[\alpha_{\nu}\right]$, and $\alpha_{\nu}(N)$ is the unique positive root of the $\nu^{\text {th }}$ order equation

$$
\frac{\Gamma\left(\alpha_{\nu}+\nu+1\right)}{\Gamma(\alpha+1)}=\nu ! N, \quad \text { and } \quad b_{\nu}(N)=N-\frac{\Gamma(k+\nu+1)}{\Gamma(k+1) \Gamma(\nu+1)} .
$$

Considerations analogous to Corollary 5.1 and subsequent paragraphs lead to bounds for $g_{\nu}(N)$ :

$$
\begin{aligned}
& \frac{\nu(\nu-1)}{24 \nu !}(\nu ! N)^{1-1 / \nu}+\frac{\nu(\nu-1)\left(\nu^{2}-9\right)}{1920 \nu !}(\nu ! N)^{1-3 / \nu}+O(\nu ! N)^{1-5 / \nu} \\
& \leq g_{\nu}(N)-\frac{\nu}{(\nu+1) !}(\nu ! N)^{1+1 / \nu}+\frac{\nu}{2} N \\
& \leq \frac{\nu(\nu+2)}{24 \nu !}(\nu ! N)^{1-1 / \nu} \\
& \quad+\frac{\nu(\nu-1)(\nu+2)(\nu+14 / 3)}{1920 \nu !}(\nu ! N)^{1-3 / \nu}+O(\nu ! N)^{1-5 / \nu}
\end{aligned}
$$


As an example, let us consider the cases $\nu=1,2$ separately. Here, the bounds need not to be expanded in powers of $N$ but can easily be given explicitely. For particles living on a plane, we get

$$
\frac{2 N}{3} \sqrt{2 N+1 / 4}-N \leq g_{2}(N) \leq \frac{1}{3} \sqrt{(2 N+1 / 3)^{3}}-N
$$

whereas for $\nu=1$ we get the trivial result

$$
g_{1}(N)=\frac{1}{2} N(N-1) .
$$

\section{References}

1. Abott, P.C., Maslen, E.N.: Coordinate systems and analytic expansions for three-body atomic wave functions. I. Partial summation for the Fock expansion in hyperspherical coordinates. J. Phys. A 20, 2043-2075 (1987)

2. Aizenman, M., Simon, B.: Brownian motion and Harnack's inequality for Schrödinger operators. Commun. Pure Appl. Math. 35, 209-237 (1982)

3. Avery, J.: Hyperspherical harmonics: applications in quantum theory. Dordrecht: Kluwer Academic Publishers 1989

4. Gilbarg, D., Trudinger, N.S.: Elliptic partial differential equations, $2^{\text {nd }}$ ed. Berlin, Heidelberg, New York: Springer 1983

5. Hinz, A.M., Kalf, H.: Subsolution estimates and Harnack's inequality for Schrödinger operators. J. Reine Angew. Math. 404, 118-134 (1990)

6. Hoffmann-Ostenhof, M., Hoffmann-Ostenhof, T.: Local properties of solutions of Schrödinger equations. Commun. Partial Diff. Eq. 17, 491-522 (1992)

7. Hoffmann-Ostenhof, M., Hoffmann-Ostenhof, T., Stremnitzer, H.: Electronic wave functions near coalescence points. Phys. Rev. Lett. 68, 3857-3860 (1992)

8. Hoffmann-Ostenhof, M., Seiler, R.: Cusp conditions for eigenfunctions of $N$-electron systems. Phys. Rev. A 23, 21-23 (1981)

9. Hörmander, L.: Uniqueness theorem for second order elliptic differential equations. Commun. Partial Diff. Eq. 8, 21-64 (1983)

10. Judd, B.R.: Operator techniques in atomic spectroscopy. New York: McGraw-Hill 1963

11. Kato, T.: On the eigenfunctions of many particle systems in quantum mechanics. Commun. Pure and Appl. Math. 10, 151-171 (1957)

12. Kato, T.: Schrödinger operators with singular potentials. Israel J. Math. 13, 135-148 (1973)

13. Kutzelnigg, W., Klopper, W.: Wave functions with terms linear in the interelectronic coordinates to take care on the correlation cusp. I. General theory. J. Chem. Phys. 94, 1985-2001 (1991)

14. Leray, J. In: Ciarlet, P.G., Roseau, M. (eds.): Trends and applications of pure mathematics to mechanics. Lecture Notes in Physics, 195, pp. 235-247. Berlin, Heidelberg, New York: Springer 1985

15. Morgan, J.D. III: Convergence properties of Fock's expansion for $S$-state eigenfunctions of the He-Atom. Theor. Chim. Acta 69, 181-223 (1986)

16. Reed, M., Simon, B.: Methods of modern mathematical physics. II. Fourier analysis, selfadjointness. New York: Academic Press 1975

17. Simon, B.: Schrödinger semigroups. Bull. Am. Math. Soc. 7, 447-526 (1982)

18. Stein, E.M., Weiss, G.: Fourier analysis on Euclidean spaces. Princeton, NJ: Princeton University Press 1971

Communicated by B. Simon 
